\title{
The rheological behavior of concentrated colloidal dispersions
}

\author{
John F. Brady \\ Division of Chemistry and Chemical Engineering, California Institute of Technology, Pasadena, \\ California 91125
}

(Received 3 February 1993; accepted 16 March 1993)

\begin{abstract}
A simple model for the rheological behavior of concentrated colloidal dispersions is developed. For a suspension of Brownian hard spheres there are two contributions to the macroscopic stress: a hydrodynamic and a Brownian stress. For small departures from equilibrium, the hydrodynamic contribution is purely dissipative and gives the high-frequency dynamic viscosity. The Brownian contribution has both dissipative and elastic parts and is responsible for the viscoelastic behavior of colloidal dispersions. An evolution equation for the pair-distribution function is developed and from it a simple scaling relation is derived for the viscoelastic response. The Brownian stress is shown to be proportional to the equilibrium radial-distribution function at contact, $g(2 ; \phi)$, divided by the short-time self-diffusivity, $D_{0}^{s}(\phi)$, both evaluated at the volume fraction $\phi$ of interest. This scaling predicts that the Brownian stress diverges at random close packing, $\phi_{m}$, with an exponent of -2 , that is, $\eta_{0}^{\prime} \sim \eta\left(1-\phi / \phi_{m}\right)^{-2}$, where $\eta_{0}^{\prime}$ is the steady shear viscosity of the dispersion and $\eta$ is the viscosity of the suspending fluid. Both the scaling law and the predicted magnitude are in excellent accord with experiment. For viscoelastic response, the theory predicts that the proper time scale is $a^{2} / D_{0}^{s}$, where $a$ is the particle radius, and, when appropriately scaled, the form of the viscoelastic response is a universal function for all volume fractions, again in agreement with experiment. In the presence of interparticle forces there is an additional contribution to the stress analogous to the Brownian stress. When the length scale characterizing the interparticle forces is comparable to the particle size, the theory predicts that there is only a quantitative contribution from the interparticle forces to the stress; the qualitative behavior, particularly the singular scaling of the viscosity and the form of the viscoelastic response, remains unchanged from the Brownian case. For strongly repulsive interparticle forces characterized by a length scale $b(>a)$, however, the theory predicts that the viscosity diverges at the random close packing volume fraction, $\phi_{b m}$, based on the length scale $b$, with a weaker exponent of -1 . The viscoelastic response now occurs on the time scale $b^{2} / D_{0}^{s}(\phi)$, but is of the same form as for Brownian dispersions.
\end{abstract}

\section{INTRODUCTION}

Colloidal dispersions occur in a variety of natural and man-made settings. An understanding of their equilibrium and transport properties is necessary for the successful exploitation of these materials, and also provides a challenging theoretical problem in the statistical mechanics of complex fluids. In this paper we consider the simplest colloidal dispersion-a suspension of monodisperse hard spheres where the particles interact through hydrodynamic and Brownian forces-and study theoretically the rheological response of these dispersions for small departures from equilibrium. There have been a number of prior studies of this problem, ${ }^{1-5}$ but no satisfactory predictions of the rheological response, particularly for concentrated dispersions, have emerged.

In this work, we make use of a new expression for the macroscopic stress in a suspension that I derived in a consideration of the hydrodynamic origin of the osmotic pressure of colloidal dispersions. ${ }^{6}$ In this new expression for the stress, the Brownian contribution is recast into two terms. The first is an integral over the surface of contact of two particles: $-n^{2} k T a \int \mathrm{nng}(\mathrm{r}) d S$, where $a$ is the particle radius, $k$ is Boltzmann's constant, $T$ is the absolute temperature, $\mathbf{n}$ is the normal along the line of centers of the two touching particles, and $g(\mathbf{r})$ is the radial-distribution func- tion. This contribution is of exactly the same form as occurs in the expression for the stress of an atomic hardsphere fluid and is present whether or not there are hydrodynamic interactions among particles. The second term in the Brownian stress is directly related to the finite size of the particles and is a result of the hydrodynamic interactions among particles [cf. Eq. (10)]. Through this new expression for the stress, we are led to make approximations for the evolution equation for the pairdistribution function which work well here, but which have not worked well with previous representations.

In the evolution equation for the pair-distribution function, two particles interact through conditionally averaged hydrodynamic interactions-that is, through hydrodynamic interactions at the concentration $\phi$, not as two particles alone at infinite dilution. This has the important consequence of showing that the relevant scaling of the pair diffusivity, which governs the diffusive response of the pair distribution to shearing deformation, is given by the short-time self-diffusivity at the concentration of interest, $D_{0}^{s}(\phi)$. This immediately shows that the characteristic time scale for response to shearing motion is $a^{2} / D_{0}^{s}(\phi)$. As shown later, and as has been noted before, ${ }^{7}$ this scaling with $D_{0}^{s}(\phi)$ collapses the viscoelastic response for all concentrations onto a single universal curve. (In a subsequent 
paper I show that this universal scaling also holds for the shear rate dependence of the viscosity far from equilibrium. ${ }^{8}$ ) Physically, it is the short-time self-diffusivity that governs the viscoelastic response because, for small departures from equilibrium, particles only need diffuse a proportionally small distance to relax the structure back to equilibrium. Large scale particle displacements, characterized by the long-time self-diffusivity, $D_{\infty}^{s}(\phi)$, are not needed to relax the near-equilibrium structure.

Under the application of a shearing motion the nondimensional parameter governing the deformation of the microstructure is the Peclet number, $\dot{\gamma} a^{2} / D_{0}^{s}(\phi)$, which can be viewed as the ratio of the diffusive relaxation time, $a^{2}$ / $D_{0}^{s}(\phi)$, to the flow time, $\dot{\gamma}^{-1}$. For weak shearing, $\dot{\gamma} a^{2} /$ $D_{0}^{s}(\phi)<1$, the perturbation to the equilibrium structure is linear in the Peclet number-the shear flow distorts the structure and this distortion is resisted by the "entropic" diffusive motion of the particles. The Brownian contribution to the deviatoric stress is proportional to the number of particles contacting each other, i.e., proportional to $g(r)$ at contact, and to the departure of the pair-distribution function from equilibrium, i.e., proportional to the Peclet number $\dot{\gamma} a^{2} / D_{0}^{s}(\phi)$. Thus, the deviatoric stress or effective viscosity scales as the radial-distribution function at contact divided by the short-time self-diffusivity, both at the volume fraction $\phi$ of interest. As random close packing is approached the number of particles "at contact" diverges as $\left(1-\phi / \phi_{m}\right)^{-1}$, and the short-time self-diffusivity vanishes as $\left(1-\phi / \phi_{m}\right)$ because the particles are "locked" into place by the strong hydrodynamic lubrication forces. Thus, the effective viscosity diverges as $\left(1-\phi / \phi_{m}\right)^{-2}$ as random close packing is approached. This is the essence behind the singular behavior of the viscosity of Brownian dispersions.

In Sec. II we develop the general equations for the evolution of the suspension microstructure and the expression for the macroscopic stress. In Sec. III we reduce the $N$-particle evolution equation to an equation for the motion of a pair and show explicitly that the relevant time scale is given by $a^{2} / D_{0}^{s}(\phi)$. We also make use of the new expression for the Brownian contribution to the stress to show explicitly the scaling of the viscosity as maximum packing is approached. In Sec. IV we solve for the perturbation to the pair-distribution function for small departures from equilibrium for a general time-dependent linear flow, and obtain explicit analytic expressions for both the real and imaginary parts of the dynamic viscosity. The viscoelastic response is shown to be a universal function of the diffusive relaxation time $a^{2} / D_{0}^{s}(\phi)$, and the predicted results are in excellent agreement with experiment for all volume fractions and all frequencies.

In Sec. $V$ we generalize the analysis and results to consider particles that interact through colloidal-scale interparticle forces in addition to Brownian and hydrodynamic forces. It is shown that when the interparticle forces are strongly repulsive, with the repulsive forces occurring on a length scale $b$ that is large compared to the hydrodynamic scale $a$, the relevant relaxation time is now $b^{2} /$ $D_{0}^{s}(\phi)$-the length scale associated with the interparticle forces divided by the short-time self-diffusivity at the vol- ume fraction based on the particle radius $a$. The interparticle force contribution to the stress is also proportional to the number of particles "at contact," which now occurs at the scale $b$, and to the departure of the structure from equilibrium, i.e., proportional to $\dot{\gamma} b^{2} / D_{0}^{s}(\phi)$. This scaling predicts that the viscosity diverges as random close packing at the volume fraction based on the length scale $b$, the "thermodynamic" volume fraction, is approached, since the number of particles at contact diverges at this value. However, the short-time self-diffusivity does not vanish as it is determined by the "hydrodynamic" volume fraction, $\phi$ based on $a$, which remains small when $b \gg a$. The particles are not "locked" by the hydrodynamic lubrication forces. Thus, the viscosity diverges with a weaker exponent of -1 . Apart from this scaling change, the viscoelastic response is identical to that for Brownian particles.

It is also shown in Sec. $V$ that when the length scale associated with the interparticle forces is of the same order as the hydrodynamic scale, both the singular scaling of the viscosity and the viscoelastic response are identical to the pure Brownian case. Finally in Sec. VI we close with a few remarks concerning the applicability of this theory to nonrandom dispersions and to the relation between the self diffusivity and the effective viscosity. It is argued that the short-time self-diffusivity scales as one over the highfrequency dynamic viscosity as close packing is approached, while the long-time self-diffusivity is proportional to one over the low-frequency dynamic or steady shear viscosity.

\section{THE PARTICLE EVOLUTION EQUATION AND SUSPENSION STRESS}

We consider a suspension of identical spherical particles of radii $a$ subjected to Brownian, interparticle and hydrodynamic forces at low Reynolds number ( $\rho \dot{\gamma} a^{2}$ / $\eta<1)$. The equation governing the distribution of particles-the microstructure-is the the well-known $N$-particle Smoluchowski equation:

$$
\frac{\partial P_{N}}{\partial t}+\nabla \cdot \mathbf{j}_{N}=0
$$

where $P_{N}$ is the probability density for the $N$ particles to be in configuration $\mathbf{x}$. The probability flux $\mathbf{j}_{N}$ is given by

$$
\mathbf{j}_{N}=\mathbf{U} P_{N}+\mathbf{R}_{F U}^{-1} \cdot\left(\mathbf{F}^{P}-k T \nabla \ln P_{N}\right) P_{N} .
$$

In Eq. (2) $U$ is the velocity of the particles due to the shear flow, and in writing this we have combined the individual particle velocity vectors (for spherical particles only the translational velocities and the positions of the centers of each particle need be considered) into a single $3 N$ vector $\mathbf{U}$. The colloidal interparticle forces are denoted by $\mathbf{F}^{P}$, and the Brownian forces are given by $k T$ times the gradient with respect to the configuration vector $\mathbf{x}$ of the log of the probability density, $-k T \nabla \ln P_{N}$. The $3 N \times 3 N$ hydrodynamic resistance tensor $\mathbf{R}_{F U}$ relates the hydrodynamic force exerted on the particles to their velocities, and its inverse, $\mathbf{R}_{F U}^{-1}$, is the $N$-particle mobility giving the velocities 
in terms of the forces. From the Stokes-Einstein relation this mobility multiplied by $k T$ is the $N$-particle diffusivity tensor:

$$
\mathbf{D}=k T \mathbf{R}_{F U}^{-1}
$$

With interparticle forces derivable from a potential, $\mathrm{F}^{P}=-\nabla V$,

the equilibrium distribution ( $U \equiv 0$ ) is given by

$$
\mathbf{j}_{N}=0 \text {, }
$$

which, since $\mathbf{D}$ is positive definite, requires that

$$
\mathbf{F}^{P}=k T \nabla \ln P_{N},
$$

whose solution is the Boltzmann distribution

$$
P_{N}^{0} \sim \exp (-V / k T) \text {, }
$$

where the superscript zero denotes equilibrium. For the case of Brownian hard spheres, the potential is infinite if the particles were to overlap and zero elsewhere, and Eq. (4) gives the well-known hard-sphere distribution.

In the presence of a shearing motion, $\mathbf{U} \neq 0$, the particle velocities are given by

$$
\mathbf{U}=(\langle\mathbf{E}\rangle+\langle\boldsymbol{\Omega}\rangle) \cdot \mathbf{x}+\mathbf{R}_{F U}^{-1} \cdot \mathbf{R}_{F E}:\langle\mathbf{E}\rangle,
$$

where $\langle\mathbf{E}\rangle$ and $\langle\boldsymbol{\Omega}\rangle$ are the bulk or macroscopic rate of strain and vorticity tensors of the imposed linear flow and are constant in spacc but may be arbitrary functions of time. $\mathbf{R}_{F E}$ is the hydrodynamic resistance tensor that gives the hydrodynamic forces on the particles due to the imposed flow. In the absence of any hydrodynamic interactions (i.e., $\mathbf{R}_{F E} \equiv 0$ ), the particles would simply be advected by the imposed linear flow: $\mathbf{U}=(\langle\mathbf{E}\rangle+\langle\boldsymbol{\Omega}\rangle) \cdot \mathbf{x}$.

The bulk stress in the suspension can be written as ${ }^{6,9-11}$

$$
\langle\Sigma\rangle=-\langle p\rangle \mathbf{I}+2 \eta\langle\mathbf{E}\rangle+\left\langle\Sigma_{p}\right\rangle
$$

where $\langle p\rangle$ is a constant setting the level of the pressure, and $2 \eta\langle E\rangle$ is the deviatoric stress contribution from the fluid, with $\eta$ the viscosity of the suspending fluid. The particle contribution to the stress $\left\langle\Sigma_{P}\right\rangle$ is given by

$$
\left\langle\Sigma_{P}\right\rangle=-n k T \mathbf{I}+n\left[\left\langle\mathbf{S}^{H}\right\rangle+\left\langle\mathbf{S}^{B}\right\rangle+\left\langle\mathbf{S}^{P}\right\rangle\right] .
$$

Here, $-n k T I$ is just the isotropic stress associated with the thermal kinetic energy of the Brownian particles, $I$ is the isotropic tensor and $n$ is the number density of particles. The hydrodynamic, $\mathbf{S}^{H}$, Brownian, $\mathbf{S}^{B}$, and interparticle, $\mathbf{S}^{\boldsymbol{P}}$, contributions to the stress are given by

$$
\begin{aligned}
& \left\langle\mathbf{S}^{H}\right\rangle=-\left\langle\mathbf{R}_{S U} \cdot \mathbf{R}_{F U}^{-1} \cdot \mathbf{R}_{F E}-\mathbf{R}_{S E}\right\rangle:\langle\mathbf{E}\rangle, \\
& \left\langle\mathbf{S}^{B}\right\rangle=-k T\left\langle\nabla \cdot\left(\mathbf{R}_{S U} \cdot \mathbf{R}_{F U}^{-1}\right)\right\rangle, \\
& \left\langle\mathbf{S}^{P}\right\rangle=-\left\langle\left(\mathbf{x I}+\mathbf{R}_{S U} \cdot \mathbf{R}_{F U}^{-1}\right) \cdot \mathbf{F}^{P}\right\rangle .
\end{aligned}
$$

The additional hydrodynamic resistance tensors, $\mathbf{R}_{S U}$ and $\mathbf{R}_{S E}$, relate the particle stresslets (symmetric first moment of the force distribution integrated over the particle surface, which gives the stress) to the particles' velocities and the rate of strain, respectively. The tensors $\mathbf{R}_{S U}$ and $\mathbf{R}_{S E}$ here are not taken to be traceless in their first two indices as has been the convention in the past. The trace of these functions can be used to determine the osmotic pressure [cf. Eq. (11)]. From the symmetry of the low-Reynolds number resistance tensors, $\mathbf{R}_{S U}$ is equal to the transpose of $\mathbf{R}_{F E}$. The angle brackets \langle\rangle denote an average over the distribution $P_{N}$. We have also assumed that there are no external torques acting on the particles, which would give rise to an antisymmetric contribution to the bulk stress of the form

$$
-n \epsilon \cdot\left\langle\mathbf{L}^{P}\right\rangle,
$$

where $\left\langle\mathbf{L}^{P}\right\rangle$ is the average external torque acting on the particles and $\epsilon$ is the unit alternating tensor. ${ }^{6}$

To determine the viscoelastic response of the dispersion, we need to solve the evolution equation (1) for $P_{N}$ and then use this in Eqs. (6)-(8c) to determine the stress. This can be carried out numerically by Stokesian dynamics simulation, ${ }^{9-12}$ but here we wish to proceed analytically. In order to do so, we first recast the Brownian contribution to the stress, $\mathbf{S}^{B}$ in Eq. (8b), into an alternate form weighted by the probability density $P_{N}$ and, in so doing, obtain the new representation for the stress I derived earlier. ${ }^{6}$ The details of this derivation can be found in the Appendix.

In place of Eq. (7) we have the following expression for the particle stress

$$
\begin{aligned}
\left\langle\Sigma_{P}\right\rangle= & -n k T \mathbf{I}-n k T a \int \mathbf{n}_{2} \mathbf{n}_{2} P_{1 / 1}\left(\mathbf{r}_{2} \mid \mathbf{r}_{1}\right) d S_{2} \\
& -n\left\langle\mathbf{x} \mathbf{F}^{P}\right\rangle-n\left\langle\mathbf{R}_{S U} \cdot \mathbf{R}_{F U}^{-1} \cdot \mathbf{R}_{F E}-\mathbf{R}_{S E}\right\rangle:\langle\mathbf{E}\rangle \\
& -n\left\langle\mathbf{R}_{S U} \cdot \mathbf{R}_{F U}^{-1} \cdot\left[\mathbf{F}^{P}-k T \nabla \ln P_{N}\right]\right\rangle,
\end{aligned}
$$

where, $P_{1 / 1}\left(\mathbf{r}_{2} \mid \mathbf{r}_{1}\right)$ is the probability density for finding a particle at $\mathbf{r}_{2}$ given that there is a particle at $\mathbf{r}_{1}$, and the integral is over the surface of contact of the two touching particles; $\mathbf{n}_{2}$ is the unit normal along the line of centers from particle 1 to 2 . In a statistically homogeneous dispersion, $P_{1 / 1}\left(\mathbf{r}_{2} \mid \mathbf{r}_{1}\right)=P_{1 / 1}(\mathbf{r})=n g(\mathbf{r})$, where $\mathbf{r}=\left(\mathbf{r}_{2}-\mathbf{r}_{1}\right)$, and $g(r)$ is the radial-distribution function.

Equation (10) is identical to Eq. (7) and is the exact form for the particle stress both in and out of equilibrium. At equilibrium, $\langle\mathbf{E}\rangle=\langle\mathbf{\Omega}\rangle=0, \mathbf{F}^{P}=k T \nabla \ln P_{N}$, and Eq. (10) reduces to the osmotic pressure of a colloidal dispersion. ${ }^{6}$ The osmotic pressure, $\Pi$, is defined, mechanically, to be minus one third the trace of $\left\langle\Sigma_{p}\right\rangle$ :

$$
\mathbf{I}:\left\langle\Sigma_{P}\right\rangle=-3 \Pi
$$

or

$$
\frac{\Pi}{n k T}=1+4 \phi g(2 a)+\frac{1}{3}\left\langle\mathbf{x} \cdot \mathbf{F}^{p} / k T\right\rangle,
$$

where $\phi=4 \pi a^{3} n / 3$ is the volume fraction of particles. The Brownian contribution to the osmotic is given by the equilibrium radial-distribution function at contact $g(r=2 a)$, and the interparticle forces give the familiar $\left\langle\mathbf{x} \cdot \mathbf{F}^{P}\right\rangle$ pressure.

Equation (10) for the particle stress shows that there are five contributions to the particle stress. The first term, $-n k T I$, is the usual kinetic one. In the second term, part of the direct Brownian contribution to the stress, $\mathbf{S}^{B}$, has been transferred to knowledge of $g(\mathbf{r})$ at contact, as is the 
case in equilibrium. Note that no hydrodynamic functions appear here. All that is needed is knowledge of how the contact value of $g(\mathbf{r})$ evolves as the shear rate changes. This term is identical to that arising from a hard-sphere potential in a molecular liquid.

In the third term part of the interparticle force contribution, $\mathbf{S}^{P}$, has been cast into the familiar $\left\langle\mathbf{x F}^{P}\right\rangle$ stress of the same form as in molecular liquids. Note that $\left\langle\mathbf{x} \mathbf{F}^{P}\right\rangle$ need not be symmetric, and, in general, will not be if the interparticle forces are noncentral. In molecular fluids most interparticle forces are central, but for colloidal particles noncentral forces can easily arise as, for example, in the case of electrorheological fluids. ${ }^{13}$ Moreover, if there are external torques acting on the particles, the antisymmetric stress from Eq. (9) must be added to Eq. (10).

The fourth term is the hydrodynamic contribution to the stress, $\mathbf{S}^{H}$ in Eq. (8a). This term is purely hydrodynamic in origin and has no counterpart in a molecular fluid. It depends fundamentally on the finite size of the particles in that the resistance tensors $\mathbf{R}_{S U}$ and $\mathbf{R}_{S E}$ are proportional to $a^{3}$; for point particles these functions are zero. For a single isolated sphere, $\mathbf{R}_{S U}=0$ and $\left|\mathbf{R}_{S E}\right|$ $=\frac{20}{3} \pi \eta a^{3}$, which, when combined with $2 \eta\langle\mathbf{E}\rangle$ for the solvent, gives the well-known Einstein viscosity $\eta_{\text {eff }}=\eta(1$ $\left.+\frac{5}{2} \phi\right)$. As we shall see later, the high-frequency dynamic viscosity of a colloidal dispersion is given by this term with the average taken over the equilibrium structure.

The final term in Eq. (10) gives the contribution to the particle stress due to the hydrodynamic stresses generated in the fluid resulting from the motion caused by the interparticle $\left(\mathbf{F}^{P}\right)$ and Brownian $\left(-k T \nabla \ln P_{N}\right)$ forces. Again, these contributions are completely hydrodynamic in origin, are due to the finite size of the particles (proportional to $a^{3}$ ), and have no counter part in molecular liquids.

Equation (10) is a reformulation for the particle stress that we shall use to study the rheological behavior of colloidal dispersions. It is important to note that with the use of hydrodynamic interactions, the interparticle force that appears in Eqs. (2) and (10) is of colloidal origin, e.g., electrostatic, steric, etc. With hydrodynamic interactions, a hard-sphere potential plays no dynamical role; it does not contribute to the evolution of particle positions nor to the macroscopic stress. This can be appreciated by noting that for a hard-sphere potential, the interparticle force is given by

$$
\mathbf{F}^{P}=\frac{1}{2} \mathbf{n} \delta(r-2 a),
$$

where $\mathbf{n}$ is the normal vector along the line of centers of the two touching particles and $\delta$ is the delta function at the surface of contact. In the flux expression Eq. (2) the interparticle force is always multiplied by the relative mobility of the two particles, $\mathbf{R}_{F U}^{-1}$, and, since the relative mobility goes to zero as the particles come into contact, the hard-sphere force causes no motion. In a like fashion, the interparticle force contribution to the bulk stress, $\mathbf{S}^{P}$ in Eq. $(8 \mathrm{c})$, is also zero. This can easily be seen by noting that with Eq. (12) for $\mathbf{F}^{P}$ the analysis in the appendix leading to Eq. (A4) holds and this term will cancel identically the $\left\langle\mathbf{x} \mathbf{F}^{P}\right\rangle$ interparticle stress. Evidently the hard-sphere na- ture of the particles is accounted for by the no-slip hydrodynamic boundary condition and the direct Brownian contribution to the stress gives the expected "collisional" contribution to the macroscopic stress. All of the previous expressions are valid for particles of any size or shape and can be easily extended to any distribution of sizes and/or shapes.

\section{PAIR-EVOLUTION EQUATION FOR SMALL DEPARTURES FROM EQUILIBRIUM AND THE SCALING OF THE SUSPENSION STRESS}

The equations in the preceding section for the $N$-particle distribution function and the stress are exact. In order to make analytical progress, we need to derive an equation for the pair-distribution function $P_{2}$. This can be found by integrating Eq. (1) over $N-2$ particles, which results in the colloidal equivalent of the BBGKY hierarchy. Such a hierarchy needs to be closed, because threebody terms arise from the many-body hydrodynamic interactions.

First, note that at equilibrium the flux $\mathbf{j}_{\alpha}$ of each and every particle $\alpha$ is identically zero. This is so because $\mathbf{F}_{\alpha}^{P}$ $=-\nabla_{\alpha} V=k T \nabla_{\alpha} \ln P_{N}^{0}$ [cf. Eq. (2)]. Departures from equilibrium may be conveniently expressed as

$$
P_{N}=P_{N}^{0}\left(1+f_{N}\right),
$$

and the particle flux Eq. (2) becomes

$$
\mathbf{j}_{N}=\mathbf{U} \boldsymbol{P}_{N}-P_{N}^{0} \mathbf{D} \cdot \nabla f_{N},
$$

where we have made use of the Stokes-Einstein relation Eq. (3). Integrating Eq. (1) over $N-2$ particles and using the homogeneity of the dispersion to note that $\nabla_{2}=-\nabla_{1}$ $=\nabla_{r}$, the evolution equation for particle pairs becomes

$$
\begin{aligned}
& \frac{\partial P_{2}}{\partial t}+\nabla_{r} \cdot\left\langle\mathbf{U}_{r}\right\rangle_{2} P_{2}-\nabla_{r} \cdot P_{2}^{0}\left\langle\mathbf{D}_{r} \cdot \nabla_{r} f_{N}\right\rangle_{2}^{0} \\
& \quad-\nabla_{r} \cdot P_{2}^{0} \int\left\langle\left(\mathbf{D}_{23}-\mathbf{D}_{13}\right) \cdot \nabla_{3} f_{N}\right\rangle_{3}^{0} P_{1 / 2}^{0}\left(\mathbf{r}_{3} \mid \mathbf{r}_{1}, \mathbf{r}_{2}\right) d \mathbf{r}_{3}=0 .
\end{aligned}
$$

In Eq. (15) $\mathbf{U}_{r}=\mathbf{U}_{2}-\mathbf{U}_{1}$ is the relative velocity of two particles arising from the imposed shearing motion, $\mathbf{D}_{r}$ is their relative diffusivity $\left(\mathbf{D}_{r}=\mathbf{D}_{22}+\mathbf{D}_{11}-\mathbf{D}_{12}-\mathbf{D}_{21}\right)$, and $\nabla_{r}$ is the gradient with respect to $\mathbf{r}=\mathbf{r}_{2}-\mathbf{r}_{1}$.

The angle brackets \langle\rangle$_{2}$ denote a conditional average with two particles fixed

$$
\begin{aligned}
\left\langle\mathbf{U}_{r}\left(\mathbf{r}_{1}, \mathbf{r}_{2}\right)\right\rangle_{2} \equiv & \frac{1}{(N-2) !} \int \mathbf{U}_{r}\left(\mathbf{r}_{1}, \mathbf{r}_{2}, \ldots, \mathbf{r}_{N}\right) \\
& \times P_{N-2 / 2}\left(\mathbf{r}_{3}, \ldots, \mathbf{r}_{N} \mid \mathbf{r}_{1}, \mathbf{r}_{2}\right) d \mathbf{r}_{3}, \ldots, \mathbf{r}_{N} .
\end{aligned}
$$

$P_{N-2 / 2}$ is the conditional probability for finding particles at $\mathbf{r}_{3}, \ldots, \mathbf{r}_{N}$ given that there are two particles at $\mathbf{r}_{1}$ and $\mathbf{r}_{2}$. The normalization factor $(N-2)$ ! arises because the particles are indistinguishable. The conditional average \langle\rangle$_{2}^{0}$ is the same, except it is over the equilibrium distribution $P_{N-2 / 2}^{0}$. 
$P_{1 / 2}^{0}\left(\mathbf{r}_{3} \mid \mathbf{r}_{1}, \mathbf{r}_{2}\right)$ is the conditional probability of finding a particle at $\mathbf{r}_{3}$ given that there are particles at $\mathbf{r}_{1}$ and $\mathbf{r}_{2}$.

The nonlinear averages in Eq. (15), $\left\langle\mathbf{D}_{r} \cdot \nabla_{r} f_{N}\right\rangle_{2}^{0}$, etc., need to be broken in order to derive an equation for $P_{2}$. Thus, we write

$$
\left\langle\mathbf{D}_{r} \cdot \nabla_{r} f_{N}\right\rangle_{2}^{0}=\left\langle\mathbf{D}_{r}\right\rangle_{2}^{0} \cdot \nabla_{r}\left\langle f_{2}\right\rangle_{2}^{0}+\int\left\langle\mathbf{D}_{r}^{\prime} \cdot \nabla_{r} f_{2}^{\prime}\right\rangle_{2}^{0} P_{1 / 2}^{0} d \mathbf{r}_{3},
$$

where

$$
\mathbf{D}_{r}^{\prime} \equiv \mathbf{D}_{r}-\left\langle\mathbf{D}_{r}\right\rangle_{2}^{0}
$$

and

$$
\nabla_{r} f_{2}^{\prime} \equiv \nabla_{r} f_{N}-\nabla_{r}\left\langle f_{2}\right\rangle_{2}^{0}
$$

Using Eq. (16) in Eq. (15), the pair-evolution equation becomes

$$
\begin{aligned}
& P_{2}^{0} \frac{\partial\left\langle f_{2}\right\rangle_{2}^{0}}{\partial t}+\nabla_{r} \cdot\left\langle\mathbf{U}_{r}\right\rangle_{2} P_{2}^{0}\left[1+\left\langle f_{2}\right\rangle_{2}^{0}\right] \\
& \quad-\nabla_{r} \cdot P_{2}^{0}\left\langle\mathbf{D}_{r}\right\rangle_{2}^{0} \cdot \nabla_{r}\left\langle f_{2}\right\rangle_{2}^{0} \\
& \quad=\nabla_{r} \cdot P_{2}^{0} \int\left\langle\mathbf{D}_{r}^{\prime} \cdot \nabla_{r} f_{2}^{\prime}+\left(\mathbf{D}_{23}-\mathbf{D}_{13}\right) \cdot \nabla_{3} f_{N}\right\rangle_{3}^{0} P_{1 / 2}^{0} d \mathbf{r}_{3} .
\end{aligned}
$$

The boundary conditions to be satisfied by the pairevolution equation are no relative flux at the surface on contact of the two particles:

$$
\begin{aligned}
\mathbf{n} \cdot\left\langle\mathbf{D}_{r}\right\rangle_{2}^{0} \cdot \nabla_{r}\left\langle f_{2}\right\rangle_{2}^{0}-\mathbf{n} \cdot\left\langle\mathbf{U}_{r}\right\rangle_{2}\left[1+\left\langle f_{2}\right\rangle_{2}^{0}\right] \\
\quad=\int \mathbf{n} \cdot\left\langle\mathbf{D}_{r}^{\prime} \cdot \nabla_{r} f_{2}^{\prime}+\left(\mathbf{D}_{23}-\mathbf{D}_{13}\right) \cdot \nabla_{3} f_{N}\right\rangle_{3}^{0} P_{1 / 2}^{0} d \mathbf{r}_{3},
\end{aligned}
$$

with $\mathbf{n}$ the normal connecting the two particles, and the perturbation should vanish at large separations:

$$
\left\langle f_{2}\right\rangle_{2}^{0} \sim 0 \text { as } r \rightarrow \infty \text {. }
$$

Equation (17) is an exact equation for the perturbation to the equilibrium pair-distribution function $\left\langle f_{2}\right\rangle_{2}^{0}$. It is coupled to higher distribution functions, $f_{3}$, etc., through the hydrodynamic interactions implicit in $\mathbf{D}$ and through the perturbation to the distribution function $f_{2}^{\prime}$. Thus, the hierarchy of equations must be truncated by a closure, and we shall make the simplest possible closure-neglect the couplings on the right hand sides (rhs) of Eqs. (17) and (18).

Departures of structure from equilibrium are driven by the hydrodynamic shearing motion, and when the shearing forces are weak, these departures may be obtained as a regular perturbation expansion about the equilibrium state. Nondimensionalization of $\mathbf{r}$ by the particle size $a$; the time by the diffusive time $a^{2} / D_{0}$, where $D_{0}=k T / 6 \pi \eta a$ is the diffusivity of a single isolated particle; the relative velocity by $\dot{\gamma} a$, where $\dot{\gamma}$ is the magnitude of the shear rate; and the introduction of the normalized disturbance

$$
\left\langle f_{2}\right\rangle_{2}^{0}=\operatorname{Pe} f_{2} \text {, }
$$

where the Peclet number measuring the relative importance of shear to Brownian forces is given by $P e=a^{2} \dot{\gamma} / D_{0}$ $=6 \pi \eta a^{3} / k T$; Eq. (17) becomes to leading order in $P_{e}$

$$
P_{2}^{0} \frac{\partial f_{2}}{\partial t}-\nabla_{r} \cdot\left\langle\mathbf{D}_{r}\right\rangle_{2}^{0} P_{2}^{0} \cdot \nabla_{r} f_{2}=-\nabla_{r} \cdot\left\langle\mathbf{U}_{r}\right\rangle_{2}^{0} P_{2}^{0} .
$$

Note that to leading order in $P e$ the average of the relative velocity is now over the equilibrium distribution $\left\langle\mathbf{U}_{r}\right\rangle_{2}^{0}$. The boundary conditions become

$$
\mathbf{n} \cdot\left\langle\mathbf{D}_{r}\right\rangle_{2}^{0} \cdot \nabla_{r} f_{2}=\mathbf{n} \cdot\left\langle\mathbf{U}_{r}\right\rangle_{2}^{0} \text { at } r=2
$$

and

$$
f_{2} \sim 0 \text { as } r \rightarrow \infty \text {. }
$$

From Eqs. (21)-(22) we see that $f_{2}$ is driven by the relative velocity of the two particles due to the shearing motion. The departures from equilibrium are "resisted" by the relative diffusivity of the two particles $\left\langle\mathbf{D}_{r}\right\rangle_{2}^{0}$, which tends to keep the pair distribution isotropic. Note, however, it is the relative velocity and diffusivity at the volume fraction $\phi$ that enters. $\left\langle\mathbf{U}_{r}\right\rangle_{2}^{0}$ and $\left\langle\mathbf{D}_{r}\right\rangle_{2}^{0}$ are "effective" two-body interactions; they are averaged interactions between two particles in the concentrated suspension. As the two particles become well separated $\left\langle\mathbf{D}_{r}\right\rangle_{2}^{0}$ asymptotes to $D_{0}^{s}(\phi)$ I, the short-time self-diffusivity of a particle, and not to $D_{0}$, the infinite dilution value. ${ }^{14}$ This suggests that we should have scaled time with $a^{2} / D_{0}^{s}(\phi)$ rather that with $a^{2} / D_{0}$. Similarly, the Peclet number should be $\dot{\gamma} a^{2} / D_{0}^{s}(\phi)$ rather than $\dot{\gamma} a^{2} / D_{0}$. This latter observation has the effect of multiplying $f_{2}$ by $D_{0}^{s}(\phi)$. Indeed, the form of Eqs. (21) $-(22)$ shows that if we rescale time, the relative diffusivity and $f_{2}$ by $D_{0}^{s}(\phi)$ according to

$$
\begin{aligned}
& \left\langle\mathbf{D}_{r}\right\rangle_{2}^{0}=\left\langle\overline{\mathbf{D}}_{r}\right\rangle_{2}^{0} D_{0}^{s}(\phi), \\
& t=\bar{t} / D_{0}^{s}(\phi), \\
& f_{2}=\bar{f}_{2} / D_{0}^{s}(\phi),
\end{aligned}
$$

then, Eqs. (21)-(22) become

$$
\begin{aligned}
& P_{2}^{0} \frac{\partial \bar{f}_{2}}{\partial \bar{t}}-\nabla_{r} \cdot\left\langle\overline{\mathbf{D}}_{r}\right\rangle_{2}^{0} P_{2}^{0} \cdot \nabla_{r} \bar{f}_{2}=-\nabla_{r} \cdot\left\langle\mathbf{U}_{r}\right\rangle_{2}^{0} P_{2}^{0}, \\
& \bar{f}_{2}-0 \quad \text { as } r \rightarrow \infty, \\
& \mathbf{n} \cdot\left\langle\overline{\mathbf{D}}_{r}\right\rangle_{2}^{0} \cdot \nabla_{r} \bar{f}_{2}=\mathbf{n} \cdot\left\langle\mathbf{U}_{r}\right\rangle_{2}^{0} \text { at } r=2 .
\end{aligned}
$$

Note that the rhs forcing in Eq. (25a) remains unchanged as $\left\langle\mathbf{U}_{r}\right\rangle_{2}^{0}$ properly asymptotes to the bulk straining, $\langle\mathbf{E}\rangle \cdot \mathbf{r}$, for large $r$. Note also that diffusivities appearing in equations are all nondimensionalized with respect to $D_{0}=k T$ / $6 \pi \eta a$, while those is the text are assumed to have units of $D_{0}$.

This simple observation of rescaling with $D_{0}^{s}(\phi)$ has far reaching consequences. It immediately shows that the appropriate time scale for viscoelastic response is $a^{2}$ / $D_{0}^{s}(\phi)$, which we shall see below collapses the response for all volume fractions onto a universal curve. This was hinted at in the work by van der Werff et al., ${ }^{7}$ where they empirically found a volume fraction-dependent time scale that collapsed their oscillatory viscosity measurements and 
recognized that this time scale was most closely related to $D_{0}^{s}(\phi)$. We see here that using the short-time selfdiffusivity arises quite naturally from the pair-evolution equation. Furthermore, although we are only considering small departures from equilibrium here, in a future paper ${ }^{8}$ I show that the steady shear viscosity for large departures from equilibrium can also be collapsed onto a single universal curve for all $\phi$ with the Peclet number based on $D_{0}^{s}(\phi)$, i.e., $\overline{P e}=P e / D_{0}^{s}(\phi)=\dot{\gamma} a^{2} / D_{0}^{s}(\phi)$. Finally, the appropriatencss of scaling with the short-time sclfdiffusivity is not affected by any of the terms we neglected in closing the hierarchy.

Values of $D_{0}^{s}(\phi)$ have been measured experimentally $^{15,16}$ and determined by Stokesian dynamics; ${ }^{17,18}$ the agreement between experiment and the numerical calculations is excellent. The analytical predictions of Beenakker and Mazur $^{19}$ are in good agreement up to $\phi \approx 0.4$. We shall take $D_{0}^{s}(\phi)$ to be know here.

The final element needed is an expression for the stress averaged down to two particles. For the moment we shall restrict our analysis to hard spheres, so $\mathbf{F}^{P} \equiv 0$ and $\mathbf{S}^{P} \equiv 0$, and return to interparticle forces later. In equilibrium the particle contribution to the stress is purely isotropic and gives the osmotic pressure Eq. (11). For small departures from equilibrium, there are contributions from the hydrodynamic stress $\mathbf{S}^{H}$ and the Brownian stress $\mathbf{S}^{B}$, both of which will be purely deviatoric; perturbations to the osmotic pressure are $o(P e)$ and must be found from the next term in the expansion in small $P e$. Since the hydrodynamic stress is already proportional to $\langle\mathbf{E}\rangle$ (or $\dot{\gamma}$ ), at leading order in $P e$ the average implied in $\mathrm{Eq}$. (8a) is over the equilibrium distribution $P_{N}^{0}$, which is just the hard-sphere distribution. Scaling this stress viscously by $\eta \dot{\gamma}$, the resistance tensor $\mathbf{R}_{S E}$ by $20 \pi \eta a^{3} / 3$, etc., we have

$$
\begin{aligned}
\left\langle\Sigma_{P}^{H}\right\rangle & \equiv-\frac{5}{2}\left\langle\mathbf{R}_{S U} \cdot \mathbf{R}_{F U}^{-1} \cdot \mathbf{R}_{F E}-\mathbf{R}_{S E}\right\rangle^{0}:\langle\mathbf{E}\rangle \\
& =\eta_{H}(\phi)\langle\mathbf{E}\rangle .
\end{aligned}
$$

The symmetry properties of the isotropic hard-sphere distribution result in a single scalar viscosity coefficient, and Eq. (26) serves to define the hydrodynamic viscosity $\eta_{H}$. In the dilute limit $\eta_{H} \sim \frac{5}{2} \phi$, which is the Einstein viscosity correction. For higher $\phi, \eta_{H}$ has been determined by Stokesian dynamics ${ }^{17,18}$ and measured experimentally. ${ }^{7,20}$ It cannot be predicted analytically for all $\phi$, although the analysis of Beenakker ${ }^{21}$ is in reasonable agreement for $\phi<0.4$. As we shall see shortly, if a suspension is subjected to a high frequency small amplitude shearing motion, then the in phase or dissipative stress response is given by the so-called high-frequency dynamic viscosity $\eta_{\infty}^{\prime}$, which is just Eq. (26) and the contribution from the fiuid

$$
\eta_{\infty}^{\prime}=1+\eta_{H}
$$

Both the measurements and Stokesian dynamics calculations for $\eta_{\infty}^{\prime}$ are in excellent agreement for all volume fractions $\phi$. We shall take $\eta_{H}(\phi)$ to be known here.

The Brownian stress will also contribute to the deviatoric stress from the perturbation of the equilibrium distribution. Since we have recast the Brownian stress into two parts, we shall designate them $\left\langle\Sigma_{P}^{B 1}\right\rangle$ and $\left\langle\Sigma_{P}^{B 2}\right\rangle$. The first contribution comes from the pair-distribution function at contact. Nondimensionalizing as before, introducing the perturbation Eq. (20), noting that $P_{2}^{0}=n P_{1 / 1}^{0}=n^{2} g(r)$, with $g(r)$ the equilibrium radial-distribution function, and that $\left\langle\Sigma_{P}^{B 1}\right\rangle$ is to be scaled with $\eta \dot{\gamma}$, whereas $\mathbf{S}^{B}$ is proportional to $k T$, we have

$$
\left\langle\Sigma_{P}^{B 1}\right\rangle=-\frac{27}{2 \pi} \phi^{2} g(2 ; \phi) \int \mathrm{nn} f_{2} d \Omega
$$

where $d \Omega$ is the solid angle of integration. If we further note that we can replace $f_{2}$ by $\bar{f}_{2} / D_{0}^{s}(\phi)$, Eq. (28) becomes

$$
\left\langle\Sigma_{P}^{B 1}\right\rangle=-\frac{27}{2 \pi} \phi^{2} \frac{g(2 ; \phi)}{D_{0}^{s}(\phi)} \int \mathbf{n n} \bar{f}_{2} d \Omega
$$

In a similar manner the second Brownian contribution to the stress in Eq. (10) becomes

$$
\left\langle\Sigma_{P}^{B 2}\right\rangle=\frac{30}{2 \pi} \phi^{2} \frac{1}{D_{0}^{S}(\phi)} \int\left\langle\mathbf{R}_{S U} \cdot \mathbf{R}_{F U}^{-1}\right\rangle_{2}^{0} \cdot\left(\nabla \bar{f}_{2}\right) g(r) d \mathbf{r}
$$

where we have neglected conditional averages with three particles fixed arising from many-body hydrodynamic interactions as was done in closing the hierarchy to obtain Eq. (21).

Equation (29) shows immediately the singular behavior of the viscosity as close packing is approached. $\left(\bar{f}_{2}\right.$ will be seen to be negative at contact, giving rise to a positive viscosity.) For a hard-sphere suspension it is known that the radial-distribution function at contact diverges at random close packing $\phi_{m} \approx 0.63$ as $\left(1-\phi / \phi_{m}\right)^{-1}$. This can be inferred from knowledge of the singular form of the osmotic pressure ${ }^{22,23}$

$$
\frac{\Pi}{n k^{\prime} T} \sim 2.9\left(1-\phi / \phi_{m}\right)^{-1} \text { as } \phi \rightarrow \phi_{m},
$$

which from Eq. (11) gives

$$
g(2) \sim 1.2\left(1-\phi / \phi_{m}\right)^{-1} \text { as } \phi \rightarrow \phi_{m} \text {. }
$$

Furthermore, experiment ${ }^{15,16}$ and Stokesian dynamics simulation $^{12,17,18}$ show that the short-time self-diffusivity goes to zero at random close packing, and, although the data are not extremely accurate, $D_{0}^{s}(\phi)$ apparently vanishes linearly as $\phi_{m}$ is approached. From the simulation data we estimate that

$$
D_{0}^{s}(\phi) \sim 0.85\left(1-\phi / \phi_{m}\right) \quad \text { as } \phi \rightarrow \phi_{m} \text {. }
$$

Thus, since $\left\langle\Sigma_{P}^{B 1}\right\rangle$ is proportional to $g(2) / D_{0}^{s}$, we immediately conclude that

$$
\left|\left\langle\Sigma_{P}^{B 1}\right\rangle\right| \sim\left(1-\phi / \phi_{m}\right)^{-2} \text { as } \phi \rightarrow \phi_{m} \text {. }
$$

Equation (33) is the most important conclusion of this analysis. Since $\left|\left\langle\Sigma_{P}^{B 1}\right\rangle\right|$ is proportional to $g(2) / D_{0}^{s}$, and since this proportionality is not altered by any of the closure approximations we have made, the singular form predicted is quite general. The form of the stress predicted by this scaling analysis has long been used to correlate exper- 
imental data, ${ }^{7,20,23-25}$ and we see now clearly why this singular form is correct. It is simply that the Brownian stress is proportional to the departure of the pair distribution at contact from its equilibrium value. This departure is in turn proportional to the equilibrium value of $g(r)$ at contact and proportional to the magnitude of the distorting force, which, in nondimensional form, is the Peclet number $\dot{\gamma} a^{2} / D_{0}^{s}$; the shearing force is resisted by the relative diffusion of the pair, and this relative diffusivity (at all separations) goes to zero as random close packing is approached. Note also that for the departure from equilibrium to be small, as has been assumed for the linear response, $\dot{\gamma} a^{2}$ / $D_{0}^{5}(\phi)<1$, which is a very stringent requirement as close packing is approached.

The singular scaling of $\left\langle\Sigma_{P}^{B 1}\right\rangle$ as $\phi \rightarrow \phi_{m}$ also suggests that this is the dominant contribution to the stress. That it is indeed the case can be argued as follows: Simulation results ${ }^{12}$ and a consideration of the lubrication singularities in the resistance tensors show that the hydrodynamic viscosity $\eta_{H}$ scales as

$$
\eta_{H} \sim\left(1-\phi / \phi_{m}\right)^{-1} \text { as } \phi \rightarrow \phi_{m},
$$

which, while singular, is less singular than $\left\langle\Sigma_{P}^{B 1}\right\rangle .\left\langle\Sigma_{P}^{B 2}\right\rangle$ from Eq. $(30)$ is proportional to $1 / D_{0}^{s}(\phi)$, and to $g(r)$. From the $1 / D_{0}^{s}(\phi)$ we get one factor of $(1-\phi /$ $\left.\phi_{m}\right)^{-1}$. However, Eq. (30) involves an integral over all $r$ of $g(r)$, not just the value of the radial-distribution function at contact. While $g(2)$ is singular, $g(r)$ is not for other values of $r$, and since $\left\langle\mathbf{R}_{S U} \cdot \mathbf{R}_{F U}^{-1}\right\rangle_{2}^{0}$ behaves as $r-2$ as two particles approach one another, the integral in $\mathrm{Eq}$. (30) should not be singular as $\phi \rightarrow \phi_{m}$. We conclude, therefore, that

$$
\left|\left\langle\Sigma_{p}^{B 2}\right\rangle\right| \sim\left(1-\phi / \phi_{m}\right)^{-1} \text { as } \phi \rightarrow \phi_{m},
$$

coming from the factor of $1 / D_{0}^{s}(\phi)$, and that the dominant contribution to the stress is $\left\langle\Sigma_{P}^{B 1}\right\rangle$.

\section{VISCOELASTIC RESPONSE}

We now turn our attention to the viscoelastic response of concentrated hard-sphere dispersions and show that not only can the scaling be predicted, but the numerical coefficients determined from a solution of Eqs. (25a)-(25c) are in quantitative agreement with experiment. We begin by noting that because of the isotropy of the equilibrium structure, the hydrodynamic relative diffusivity and velocity depend only on the separation vector $\mathbf{r}$ and can be written as ${ }^{1,14}$

$$
\begin{aligned}
\left\langle\overline{\mathbf{D}}(\mathbf{r})_{r}\right\rangle_{2}^{0} & =2\left[\mathbf{d d}\langle\bar{G}(r)\rangle_{2}^{0}+(\mathbf{I}-\mathbf{d d})\langle\bar{H}(r)\rangle_{2}^{0}\right], \\
\left\langle\mathbf{U}_{r}\right\rangle_{2}^{0}= & r \mathbf{d} \cdot\langle\mathbf{E}\rangle-r \mathbf{d} \cdot\langle\mathbf{E}\rangle \cdot\left[\mathbf{d d}\langle A(r)\rangle_{2}^{0}+(\mathbf{I}-\mathbf{d d})\right. \\
& \left.\times\langle B(r)\rangle_{2}^{0}\right],
\end{aligned}
$$

where $\langle\bar{G}(r)\rangle_{2}^{0},\langle\bar{H}(r)\rangle_{2}^{0},\langle A(r)\rangle_{2}^{0}$, and $\langle B(r)\rangle_{2}^{0}$ are the nondimensional effective hydrodynamic interactions (parallel and perpendicular to the line of centers) between two particles in the suspension, and $\mathbf{d}$ is the dimensionless separation vector between particle centers. These functions are scaled so that $\langle\bar{G}(r)\rangle_{2}^{0}$ and $\langle\bar{H}(r)\rangle_{2}^{0}$ asymptote to 1 at large separations, while $\langle A(r)\rangle_{2}^{0}$ and $\langle B(r)\rangle_{2}^{0}$ asymptote to zero at large $r$.

Since we wish to consider viscoelastic response at frequency $\omega$, the bulk shearing motion is characterized by the time-dependent rate of strain tensor

$$
\langle\mathbf{E}\rangle=\mathbf{E} e^{i \omega t},
$$

or in dimensionless form

$$
\langle\mathbf{E}\rangle=\mathbf{E} e^{i \alpha \bar{t}},
$$

where $\alpha=\omega a^{2} / D_{0}^{s}(\phi)$ is the frequency nondimensionalized by the characteristic time $a^{2} / D_{0}^{s}(\phi)$, and $\mathbf{E}$ is a constant tensor giving the type of linear flow (simple shear, elongational flow etc.).

Finally, the scalar function $\bar{f}_{2}$ must be linear in the tensor E:

$$
\bar{f}_{2}(r, \bar{t})=-\frac{1}{2} f(r) e^{i \alpha \bar{t}} \cdot \mathbf{d} \cdot \mathbf{d} .
$$

Substituting Eqs. (39), (36), and (37) into Eqs. (25a)(25c) we obtain the equation for $f(r)$ :

$$
\begin{gathered}
-i \alpha f+\frac{1}{g r^{2}} \frac{d}{d r}\left(g r^{2}\langle\bar{G}\rangle_{2}^{0} \frac{d f}{d r}\right)-6\langle\bar{H}\rangle_{2}^{0} \frac{f}{r^{2}} \\
=-\langle W\rangle_{2}^{0}-r\left(1-\langle A\rangle_{2}^{0}\right) \frac{d \ln g}{d r},
\end{gathered}
$$

$f \sim 0$ as $r \rightarrow \infty$,

$\langle\bar{G}\rangle_{2}^{0} \frac{d f}{d r}=-2\left(1-\langle A\rangle_{2}^{0}\right) \quad$ at $r=2$,

and $\langle W\rangle_{2}^{0}$, which comes from the divergence of the relative velocity, is given by

$$
\langle W\rangle_{2}^{0}=-3\left(\langle A\rangle_{2}^{0}-\langle B\rangle_{2}^{0}\right)-r \frac{d\langle A\rangle_{2}^{0}}{d r} .
$$

Using Eq. (39) for $\bar{f}_{2}$ and noting that $\left\langle\mathbf{R}_{S U} \cdot \mathbf{R}_{F U}^{-1}\right\rangle_{2}^{0}=\left\langle\mathbf{R}_{F U}^{-1} \cdot \mathbf{R}_{F E}\right\rangle_{2}^{0}$, which can be expressed in terms of $\langle A\rangle_{2}^{0}$ and $\langle B\rangle_{2}^{0}$, we can rewrite the two Brownian contributions to the stress in terms of viscosity coefficients. That is, $\left\langle\boldsymbol{\Sigma}_{p}^{B}\right\rangle$ will be strictly proportional to $\langle\mathbf{E}\rangle=\mathbf{E} e^{i \alpha \bar{t}}$; in a linear flow, normal stress differences occur at higher order in Pe. Carrying out the necessary angular integrations in Eqs. (29) and (30) we have

$$
\eta_{B 1}=\frac{9}{5} \phi^{2} \frac{g(2)}{D_{0}^{s}} f(2)
$$

and

$$
\eta_{B 2}=\frac{9}{40} \phi^{2} \frac{1}{D_{0}^{s}} \int_{2}^{\infty}\left(r \frac{d f}{d r}\langle A\rangle_{2}^{0}+3 f\langle B\rangle_{2}^{0}\right) g(r) r^{2} d r .
$$

It should be noted that $f$ is, in general, imaginary and therefore the Brownian viscosities will have both real and imaginary parts; the real part corresponds to dissipation and the imaginary part elasticity. Thus, we can separate the frequency dependent total viscosity into real and imaginary parts: 


$$
\eta_{r}=\eta^{\prime}(\omega)-i \eta^{\prime \prime}(\omega),
$$

where

$$
\eta^{\prime}(\omega)=1+\eta_{H}+\eta_{B 1}^{\prime}+\eta_{B 2}^{\prime},
$$

and

$$
\eta^{\prime \prime}(\omega)=\eta_{B 1}^{\prime \prime}+\eta_{B 2}^{\prime \prime}
$$

$\eta_{B 1}^{\prime}, \eta_{B 1}^{\prime \prime}, \eta_{B 2}^{\prime}$, and $\eta_{B 2}^{\prime \prime}$ correspond to the real and imaginary parts of $\eta_{B 1}$ and $\eta_{B 2}$, respectively.

We first note the limits of large and small frequency. At high frequency, $\omega \rightarrow \infty$ on the scale of $a^{2} / D_{0}^{s}$, which corresponds to $\alpha \rightarrow \infty$, the solution for $f$ is simply $f=0$. The frequency is so high and the amplitude so small that the suspension is not disturbed from its equilibrium state. Thus, the Brownian contributions to the stress are zero, and the high-frequency dynamic viscosity, $\eta_{r}(\omega \rightarrow \infty)$, is purely real, and therefore dissipative, and equal to 1 plus the hydrodynamic viscosity

$$
\eta_{r}=\eta^{\prime}(\infty) \equiv \eta_{\infty}^{\prime}=1+\eta_{H} .
$$

The values of $\eta_{\infty}^{\prime}$ determined by Stokesian dynamics ${ }^{17,18}$ are in excellent agreement with experiment. ${ }^{7}$

In the other extreme of low frequency, $\omega \rightarrow 0$, we have steady shear, and in this case $f$ is purely real. There is no elastic stress but only a dissipative stress characterized by the low-frequency dynamic or steady shear viscosity, $\eta_{0}^{\prime}$,

$$
\eta_{r}=\eta^{\prime}(0) \equiv \eta_{0}^{\prime}=1+\eta_{H}+\eta_{B 1}^{\prime}(0)+\eta_{B 2}^{\prime}(0),
$$

where $\eta_{B 1}^{\prime}(0)$ and $\eta_{B 2}^{\prime}(0)$ are given by Eqs. (42) and (43) with $f$ found from Eq. (40a) with $\alpha=0$. We shall turn to the determination of $f$ shortly.

The forms of the high- and low-frequency limits of the viscosity suggest that the reduced viscosity functions:

$$
\frac{\eta^{\prime}(\omega)-\eta_{\infty}^{\prime}}{\eta_{0}^{\prime}-\eta_{\infty}^{\prime}}=\frac{\eta_{B 1}^{\prime}(\omega)-\eta_{B 1}^{\prime}(\infty)+\eta_{B 2}^{\prime}(\omega)-\eta_{B 2}^{\prime}(\infty)}{\eta_{B 1}^{\prime}(0)-\eta_{B 1}^{\prime}(\infty)+\eta_{B 2}^{\prime}(0)-\eta_{B 2}^{\prime}(\infty)}
$$

and

$$
\frac{\eta^{\prime \prime}(\omega)}{\eta_{0}^{\prime}-\eta_{\infty}^{\prime}}=\frac{\eta_{B 1}^{\prime \prime}(\omega)+\eta_{B 2}^{\prime \prime}(\omega)}{\eta_{B 1}^{\prime}(0)-\eta_{B 1}^{\prime}(\infty)+\eta_{B 2}^{\prime}(0)-\eta_{B 2}^{\prime}(\infty)},
$$

are given only by the Brownian contributions to the stress and are functions of the dimensionless frequency $\omega a^{2} / D_{0}^{s}$ and $\phi$. Furthermore, we shall see later that the reduced viscosity functions are functions of $\omega a^{2} / D_{0}^{s}$ only; the explicit $\phi$ dependence has been removed through the use of $D_{0}^{s}$ and by normalizing out the overall amplitude which depends on $\phi$.

To make further progress we must solve Eqs. (40a)(40c) for $f$. Here we make the simplest approximation possible, which at first sight seems very crude, but actually leads to accurate predictions for all $\phi$ with our new representation for the stress. We neglect all hydrodynamic interactions, setting $\langle\bar{G}(r)\rangle_{2}^{0}=\langle\bar{H}(r)\rangle_{2}^{0}=1$ and $\langle A(r)\rangle_{2}^{0}$ $=\langle B(r)\rangle_{2}^{0}=0$. Note we do this after having scaled out the short-time self-diffusivity. Furthermore, we shall simply take the equilibrium distribution function $g$ to be equal to 1 , which is only correct at infinite dilution, but this will allow us to obtain an analytical solution for $f$. These two assumptions are obviously approximations, but since we know that the dominant contribution to the stress is given by $f(2)$, these approximations turn out to be accurate. Note also that when neglecting hydrodynamic interactions the second Brownian contribution to the stress is zero, $\eta_{B 2}=0$. With these approximations, the equation for $f$ now becomes

$$
\begin{aligned}
& \frac{1}{r^{2}} \frac{d}{d r}\left(r^{2} \frac{d f}{d r}\right)-6 \frac{f}{r^{2}}-i \alpha f=0, \\
& f \sim 0 \text { as } r \rightarrow \infty, \\
& \frac{d f}{d r}=-2 \text { at } r=2,
\end{aligned}
$$

which can be solved exactly. Note that the function $f$ is forced by the boundary condition at the surface of contact $r=2$.

The solution to Eq. (51) in the limit $\alpha \rightarrow 0$ corresponding to steady shear is simply

$$
f(r)=\frac{32}{3} \frac{1}{r^{3}}
$$

which gives a value of $f(2)=\frac{4}{3}$. We note that if one solves Eqs. (40a)-(40c) with exact two-body hydrodynamics and $g=1$, as done by Batchelor, ${ }^{1}$ the value of $f$ at contact is 1.42 , which is not very different from that found here. Using $\frac{4}{3}$ in Eq. (42) for $\eta_{B 1}$ we have

$$
\eta_{B 1}=\eta_{B 1}^{\prime}(0)=\frac{12}{5} \phi^{2} \frac{g(2 ; \phi)}{D_{0}^{s}(\phi)}
$$

Thus, the zero shear-rate viscosity is given by

$$
\eta_{r}=\eta_{0}^{\prime}=1+\eta_{H}+\frac{12}{5} \phi^{2} \frac{g(2 ; \phi)}{D_{0}^{s}(\phi)} \text {. }
$$

This prediction for $\eta_{0}^{\prime}$ is shown in Fig. 1 along with the experimental data of van der Werff and de Kruif, ${ }^{20}$ Krieger, ${ }^{26}$ and the Stokesian dynamics simulations of Phung. ${ }^{12}$ The agreement over the entire range of $\phi$ is truly remarkable. In using Eq. (53) we have used the Carnahan-Starling ${ }^{27}$ equation of state for $g(2)$ :

$$
g(2)=\frac{1-\frac{1}{2} \phi}{(1-\phi)^{3}}
$$

for $\phi$ less than the phase transition value of 0.5 and the singular form Eq. (31) for $\phi>0.5$. The values of the shorttime diffusivity as a function of $\phi$ where taken from the work of $\mathrm{Ladd}^{18}$ for $\phi \leqslant 0.45$ and from the work of Phung ${ }^{12}$ for larger $\phi$. Note, with our estimate of $f(2)=\frac{4}{3}$, the singular scaling prediction for the zero shear-rate viscosity as $\phi \rightarrow \phi_{m}$ is

$$
\eta_{0}^{\prime} \sim 1.3\left(1-\phi / \phi_{m}\right)^{-2} \text { as } \phi \rightarrow \phi_{m} .
$$

Although this singular form has been used to fit experimental data in the past and, with a coefficient of 1 , has been found to be accurate over a wide range of $\phi$, from our 


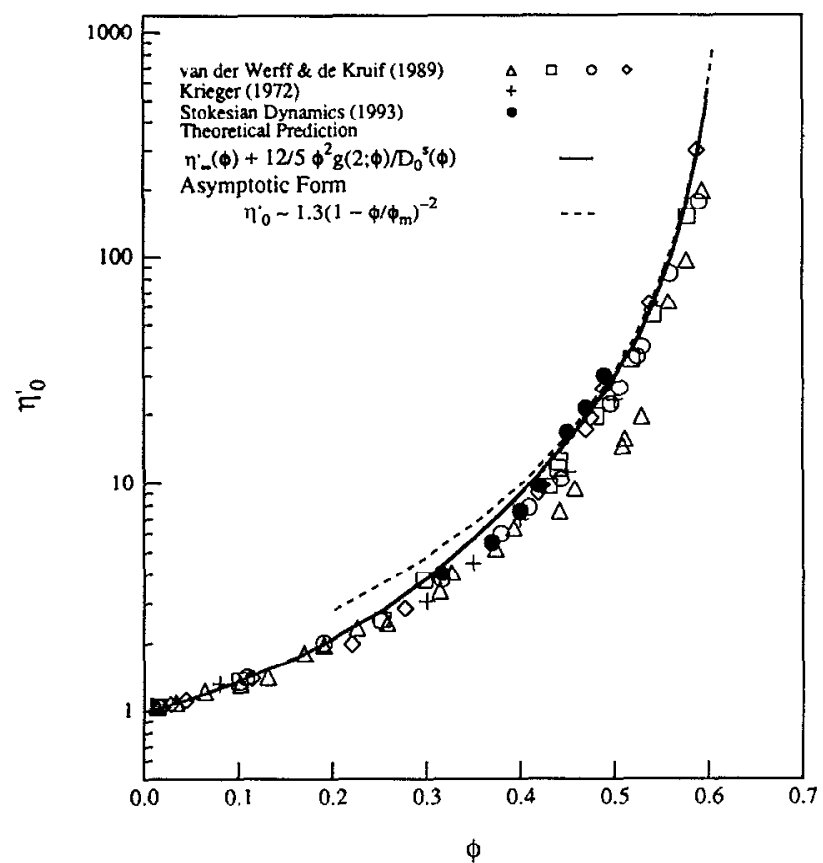

FIG. 1. Comparison of the theoretical prediction, Eq. (54) (solid line) for the zero-frequency or steady shear viscosity of Brownian hard spheres with the experimental results of van der Werff and de Kruif (Ref. 20) (open symbols), Krieger (Ref. 26) $(+)$ and the Stokesian dynamics simulations of Phung (Ref. 12) (-) The dashed line is the asymptotic prediction as random close packing, $\phi_{m}=0.63$, is approached: $\eta_{0}^{\prime}$ $\sim 1.3\left(1-\phi / \phi_{m}\right)^{-2} . D_{0}^{s}(\phi)$ has been nondimensionalized by the StokesEinstein diffusivity $D_{0}=k T / 6 \pi \eta a$.

analysis this is the correct form only as $\phi \rightarrow \phi_{m}$; for smaller $\phi$ the hydrodynamic viscosity contributes and this scales as $\left(1-\phi / \phi_{m}\right)^{-1}$ not as $\left(1-\phi / \phi_{m}\right)^{-2}$.

Equation (51) for finite $\alpha$ is recognized as Bessel's equation, and the solution is

$$
\begin{aligned}
f(z)= & \frac{2}{\sqrt{i \alpha}}\left(\frac{z_{0}}{z}\right)^{1 / 2} K_{5 / 2}(z) \\
& \times\left(K_{3 / 2}\left(z_{0}\right)+\frac{3}{z_{0}} K_{5 / 2}\left(z_{0}\right)\right)^{-1},
\end{aligned}
$$

with

$$
z=r \sqrt{i \alpha} \text { and } z_{0}=2 \sqrt{i \alpha} .
$$

Here $K_{5 / 2}$ and $K_{3 / 2}$ are the Bessel functions of half-integral order of complex argument, and they can be expressed in terms of other elementary functions. After tedious algebra $f$ can be broken up into its real and imaginary parts at contact $r=2$ :

$$
\Re f(2)=\frac{4}{3} \frac{1+2 \beta+\beta^{2}+\frac{2}{9} \beta^{3}+\frac{11}{9} \beta^{4}+\frac{4}{27} \beta^{5}}{\left(1+\beta-\frac{2}{9} \beta^{3}\right)^{2}+\beta^{2}\left(1+\frac{8}{9} \beta+\frac{2}{9} \beta^{2}\right)^{2}}
$$

and

$$
\Im f(2)=-\frac{8}{27} \frac{\beta^{2}\left(1+2 \beta+2 \beta^{2}+\frac{2}{3} \beta^{3}\right)}{\left(1+\beta-\frac{2}{9} \beta^{3}\right)^{2}+\beta^{2}\left(1+\frac{8}{9} \beta+\frac{2}{9} \beta^{2}\right)^{2}},
$$

where

$$
\beta=\sqrt{2}\left(\frac{\omega a^{2}}{D_{0}^{s}(\phi)}\right)^{1 / 2}
$$

With these expressions for the real and imaginary parts of $f(2)$ we can write the reduced forms of the real and imaginary parts of the complex viscosity as

$$
\frac{\eta^{\prime}(\omega)-\eta_{\infty}^{\prime}}{\eta_{0}^{\prime}-\eta_{\infty}^{\prime}}=\frac{3}{4} \Re f\left(2 ; \frac{\omega a^{2}}{D_{0}^{s}}\right)
$$

and

$$
\frac{\eta^{\prime \prime}(\omega)}{\eta_{0}^{\prime}-\eta_{\infty}^{\prime}}=-\frac{3}{4} \mathfrak{\Im} f\left(2 ; \frac{\omega a^{2}}{D_{0}^{s}}\right)
$$

This simple result for $\int$ predicts that the reduced viscosities should be universal functions of the reduced frequency $\omega a^{2} / D_{0}^{s}$, independent of the volume fraction $\phi$. In Fig. 2 we compare the predictions of Eqs. (59) and (60) with the experimental data of van der Werff et al. ${ }^{7}$ In the experiments the authors rescaled their frequencies with an empirically determined function of $\phi$ and noted that this function was most closely related to $D_{0}^{s}$. We do not have the raw experimental data available to us, and so have simply replotted their reported data and drawn in the predicted curves from Eqs. (59) and (60); this assumes that the cxperimentally determincd scaling for the frequency is close to $D_{0}^{s}$. From the good agreement for all frequencies, we see that the reduced viscosities are to a very good approximation universal functions of $\omega a^{2} / D_{0}^{s}$. The simple approximations capture both the qualitative and quantitative behavior for all $\phi$ and all $\omega$. The additional Brownian contribution $\eta_{B 2}$ to the viscosity is evidently small, as are the potential volume fraction dependences indicated in the full equation for $f$ through the equilibrium pair-distribution function and the effective hydrodynamic interactions.

From the analytical solution we can also determine the asymptotic form for large and small $\omega$. For small $\omega$ the departure from the zero shear-rate viscosity occurs as a regular perturbation expansion in $\alpha$ in Eq. (51). The perturbation to the pair-distribution function at contact has the asymptotic form

$$
\begin{aligned}
f(2) \sim & \frac{4}{3}\left\{1-2\left(\frac{2 \omega a^{2}}{D_{0}^{s}}\right)^{3 / 2}-i\left[\frac{2}{9}\left(\frac{2 \omega a^{2}}{D_{0}^{s}}\right)\right.\right. \\
& \left.\left.-2\left(\frac{2 \omega a^{2}}{D_{0}^{s}}\right)^{3 / 2}\right]\right\} \text { as } \frac{2 \omega a^{2}}{D_{0}^{s}} \rightarrow 0 .
\end{aligned}
$$

The first correction is purely imaginary, and therefore elastic, and linear in $\omega$. Interestingly, the first correction to the real part of $f(2)$ appears as $\omega^{3 / 2}$, a nonanalytic dependence that can be traced to the singular form of the perturbation at large $r$ reminiscent of heat transfer in steady shear flow. ${ }^{28}$ 

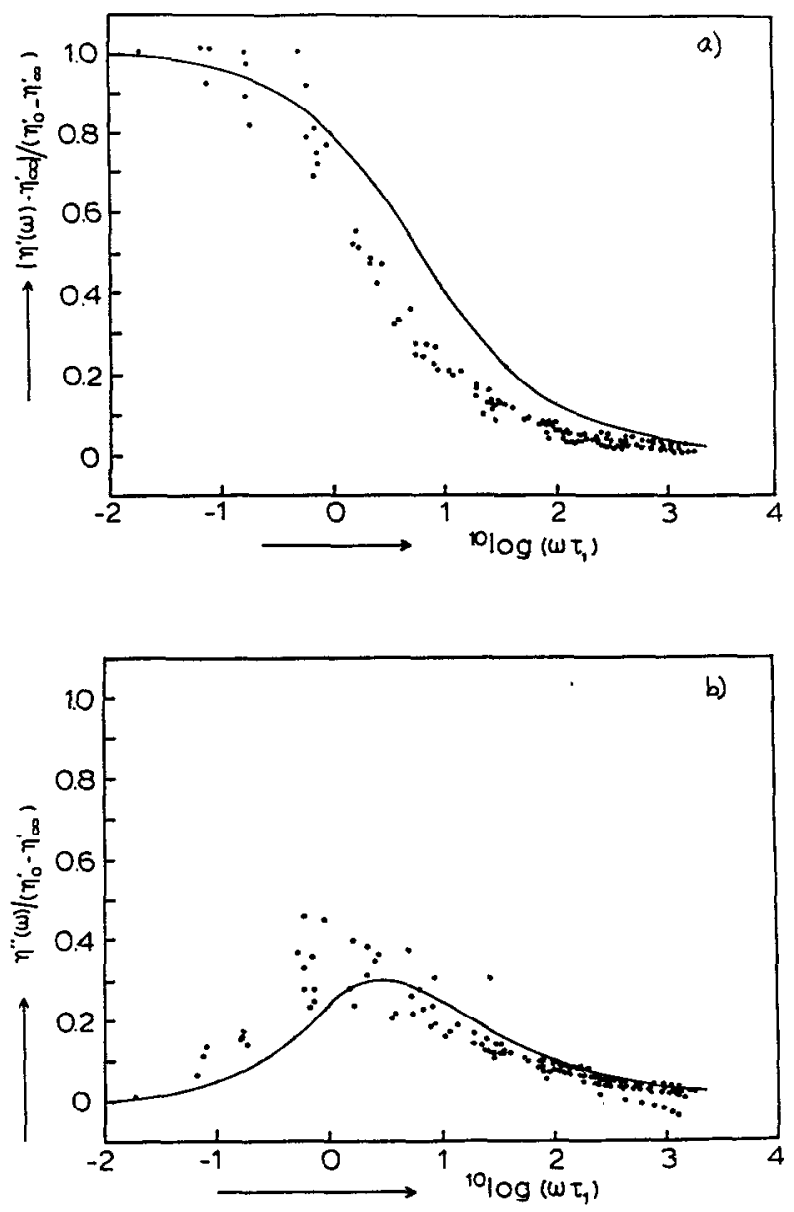

FIG. 2. Comparison of the theoretical predictions for the (a) real and (b) imaginary parts of the complex viscosity of Brownian hard spheres, Eqs. (59) and (60), with the experimental measurements of van der Werff et al. (Ref. 7). The cxperimental data were normalized by an experimentally determined time scale $\tau_{1}$, which is close, but not necessarily equal, to $a^{2} / D_{0}^{s}(\phi)$. The theoretical curves are functions of $a^{2} /$ $D_{0}^{\prime}(\phi)$ The experimental data are for the concentration range $0.46 \leqslant \phi \leqslant 0.60$.

At high frequency, $f$ asymptotes to zero, but the perturbation about infinite frequency is singular. It is easy to see from Eq. (51) that in the limit $\alpha \rightarrow \infty$ there will be a boundary layer of thickness $\alpha^{-1 / 2}$ near $r=2$ in order to satisfy the condition on the gradient of $f$. Rescaling $r$ by $y=(r-2) \sqrt{i \alpha}$ and $f$ by $f=h(y) \sqrt{i \alpha}$, in the differential equation (51) there will now be a balance between $i \alpha f$ and $d^{2} f / d r^{2}$, whose solution is

$$
h(y)=2 e^{-y}
$$

or

$$
f(r)=(2 / \sqrt{i \alpha}) e^{(i \alpha)^{1 / 2}(r-2)},
$$

giving

$$
f(2)=\left(\frac{2 D_{0}^{s}}{\omega a^{2}}\right)^{1 / 2}(1-i)
$$

Equation (62) shows that both the real and imaginary parts of the complex viscosity approach zero as $\omega^{-1 / 2}$
While this scaling with $\omega^{-1 / 2}$ apparently agrees well with the experiments of van der Werff et al. ${ }^{7}$ as seen in Fig. 2 , there is a problem with this slow decay to zero. The elastic modulus, $G^{\prime}$, of a material is related to the imaginary part of the viscosity by

$$
G^{\prime}=\omega \eta^{\prime \prime}(\omega),
$$

and, an $\eta^{\prime \prime}$ decaying as $\omega^{-1 / 2}$ indicates an elastic modulus that grows as $\omega^{1 / 2}$ as $\omega \rightarrow \infty$. An unbounded modulus is certainly not to be expected, and this aphysical behavior can be traced directly to the approximations of neglecting the equilibrium structure and the hydrodynamic interactions in Eq. (40a).

To obtain a finite modulus at high frequency, we note simply that if we retain the two terms on the rhs of Eq. (40a), then in the limit of $\alpha \rightarrow \infty$ the solution for $f$ is

$$
f(r)=-i \frac{D_{0}^{s}}{\omega a^{2}}\left(\langle W\rangle_{2}^{0}-r\left(1-\langle A\rangle_{2}^{0}\right) \frac{d \ln g}{d r}\right),
$$

which will remove the singular behavior of $G^{\prime}$. If we keep with our simple approximations, then $f(2)=2 i D_{0}^{s}$ $\omega a^{2}(d \ln g(2) / d r)$, and $\eta^{\prime \prime}(\omega)$ behaves as

$\frac{\eta^{\prime \prime}(\omega)}{\eta_{0}^{\prime}-\eta_{\infty}^{\prime}} \sim-\frac{3}{2} \frac{D_{0}^{s}}{\omega a^{2}} \frac{1}{g(2)} \frac{d g(2)}{d r}$ as $\omega a^{2} / D_{0}^{s} \rightarrow \infty$,

or, in terms of the high-frequency elastic modulus $G_{\infty}^{\prime} \equiv \lim _{\omega \rightarrow \infty} G^{\prime}(\omega)$

$$
\frac{a^{3} G_{\infty}^{\prime}}{k T}=-\frac{3}{5 \pi} \phi^{2} g(2 ; \phi) \frac{d \ln g(2 ; \phi)}{d r}=-\frac{3}{5 \pi} \phi^{2} \frac{d g(2 ; \phi)}{d r} .
$$

The high-frequency elastic modulus is proportional to the slope of the equilibrium radial-distribution function at contact: the elastic modulus is proportional to the number of particles at contact, $g(2)$, times the derivative of the "interparticle energy," $d \ln g / d r$. Note that the factors of $g(2)$ and $D_{0}^{s}$ have canceled in the nondimensional $G_{\infty}^{\prime}$. I have been unable to obtain an expression for or values of $d g / d r$ at contact (approaching from the fluid side), as this has apparently not been worked out for hard spheres. Numerically, $d g / d r$ is finite and increases in magnitude as $\phi$ increases. One expects $d g / d r$ to be singular as $\phi \rightarrow \phi_{m}$, but the exponent is unknown. Note also that $G^{\prime}$ starts off proportional to $\phi^{3}$ in the absence of hydrodynamic interactions. It is also interesting to note that Eq. (64) for $f$ is purely imaginary, indicating that the real part of $f$ will be smaller than $\omega^{-1}$; most probably scaling as $\omega^{-3 / 2}$ as $\omega \rightarrow \infty$.

The aphysical behavior of the high-frequency elastic modulus can also be removed by hydrodynamic interactions. With hydrodynamics the boundary layer at the surface of contact now scales as $\alpha^{-1}$ rather than $\alpha^{-1 / 2}$, which supports the notion of $f$ scaling as $\alpha^{-1}$. This change in boundary-layer scaling comes about because the relative diffusivity $\langle\bar{G}\rangle_{2}^{0} \sim r-2$ near $r=2$, in effect canceling one of the derivatives in Eq. (40a) for $f$. Thus, with hydrodynamics $\langle W(2)\rangle_{2}^{0}$ will determine $f$. For pairwise hydrodynamics ${ }^{1}$ with $g=1, W(2)=6.37$ and we have 


$$
\frac{\eta^{\prime \prime}(\omega)}{\eta_{0}^{\prime}-\eta_{\infty}^{\prime}} \sim \frac{3}{4} \frac{D_{0}^{s}}{\omega a^{2}}\langle W(2)\rangle_{2}^{0} \approx 4.8 \frac{D_{0}^{s}}{\omega a^{2}} \quad \text { as } \omega a^{2} / D_{0}^{s} \rightarrow \infty,
$$

or, in terms of the high-frequency elastic modulus $G_{\infty}^{\prime}$

$$
\frac{a^{3} G_{\infty}^{\prime}}{k T}=\frac{3}{10 \pi} \phi^{2} g(2 ; \phi)\langle W(2)\rangle_{2}^{0} \approx 0.61 \phi^{2} g(2 ; \phi) .
$$

With hydrodynamics we see that $G_{\infty}^{\prime}$ should scale as

$$
G_{\infty}^{\prime} \sim\left(1-\phi / \phi_{m}\right)^{-1} \text { as } \phi \rightarrow \phi_{m},
$$

which is also the scaling one might expect from the derivative of $g(r)$ at contact. Also note that with hydrodynamics $G_{\infty}^{\prime}$ starts off proportional to $\phi^{2}$ rather than $\phi^{3}$, which could serve as an experimental check on the importance of hydrodynamic interactions.

To be consistent, if we invoke $\langle W\rangle_{2}^{0}$ to remove the aphysical behavior at large $\omega$, then we would need to keep the other hydrodynamic interactions and the additional Brownian contribution to the stress $\eta_{B 2}$. This is beyond the scope of the present paper, however. In any event, these additional terms should only have a quantitative affect on the results.

As a final remark, we note that if we assume the equilibrium structure to be solidlike rather than fluidlike as we have up to now, then we can determine the zero-frequency elastic modulus $G_{0}^{\prime}$ by subjecting the material to an affine strain $\Gamma$ :

$$
P_{N}=P_{N}^{0}+\mathrm{x} \cdot \Gamma \cdot \nabla P_{N}^{0} .
$$

The Brownian stress $\left\langle\Sigma_{P}^{B 1}\right\rangle$ is the only stress that contributes (hydrodynamics cannot contribute to an equilibrium property such as the linear elastic modulus), and from Eq. (10) we have for Brownian hard spheres

$$
\frac{a^{3} G_{0}^{\prime}}{k T}=-\frac{3}{5 \pi} \phi^{2} g(2 ; \phi) \frac{d \ln g(2 ; \phi)}{d r} .
$$

Neglecting hydrodynamic interactions the high- and lowfrequency elastic moduli are identical, just as in the case of atomic solids. Differences between $G_{0}^{\prime}$ and $G_{\infty}^{\prime}$ can thus be traced to the presence of hydrodynamic interactions.

\section{EFFECTS OF INTERPARTICLE FORCES}

We now turn to a consideration of the effects of interparticle forces on the viscoelastic response of colloidal dispersions. We shall consider repulsive or weakly attractive forces such that the equilibrium state is a disordered fluid. (Even though hard spheres undergo a phase transition to an ordered state for $\phi>0.5$, we have presumed that they can be maintained in a disordered, perhaps glassy, state above this value during the viscoelastic measurements, which seems to occur in practice due to the very long relaxation times $a^{2} / D_{0}^{s}$. (Actually, the time scale for relaxation for significant departures from equilibrium is that for particles to move a significant fraction of their size, which is given by the long-time self diffusivity $D_{\infty}^{s}$. $D_{\infty}^{s}$ decreases to zero as random close packing is approached more quickly than $D_{0}^{s}$, resulting in an even longer time to relax.) Strongly attractive forces may result in a flocculated dispersion at equilibrium with a measurable yield stress and should be modeled as an elastic solid rather than as a fluid as done here. The original formulation of the problem, Eqs. (1)-(10), still holds, however, for flocculated dispersions.

With these conditions, the analysis leading to Eq. (25a) remains unchanged. Indeed, the perturbation to the pair-distribution function is still given by Eqs. (39)-(40c). In other words, all that is needed are the additional stress contributions $-n\left\langle\mathbf{x} \mathbf{F}^{P}\right\rangle$ and $-n\left\langle\mathbf{R}_{S U} \cdot \mathbf{R}_{F U}^{-1} \cdot \mathbf{F}^{P}\right\rangle$. Averaging down to two particles, nondimensionalizing as before and noting that a single scalar viscosity results, the two additional contributions to $\eta_{r}$ are

$$
\eta_{P 1}=-\frac{9}{40} \phi^{2} \frac{1}{D_{0}^{s}} \int_{2}^{\infty} r^{3} \frac{d\langle V\rangle_{2}^{0}}{d r} g(r) f(r) d r
$$

and

$$
\eta_{P 2}=\frac{9}{40} \phi^{2} \frac{1}{D_{0}^{s}} \int_{2}^{\infty} r^{3}\langle A\rangle_{2}^{0} \frac{d\langle V\rangle_{2}^{0}}{d r} g(r) f(r) d r,
$$

where the designation $\eta_{P 1}$ and $\eta_{P 2}$ follows the convention used for the Brownian stress. The conditionally averaged interparticle potential, $\langle V\rangle_{2}^{0}$, is the "potential of mean force" commonly used in statistical mechanics.

To assess the effects of interparticle forces we need simply determine $f$ and evaluate $\eta_{P 1}$ and $\eta_{P 2}$. If the variation of the equilibrium structure from the hard-sphere distribution is small everywhere (for all $r$ ), as may occur when the interparticle forces are weak, then we can use the same approximations as done in obtaining $\mathrm{Eq} .(52)$ for $f$. Thus, for uniformly small departures from the hard-sphere distribution, the zero-frequency interparticle force contribution to the viscosity is simply

$$
\eta_{P_{1}}=-\frac{24}{5} \phi^{2} \frac{1}{D_{0}^{s}} \int_{2}^{\infty} \frac{d\langle V\rangle_{2}^{0}}{d r} g(r) d r
$$

which can be integrated by parts to give

$$
\begin{aligned}
\eta_{P 1}= & \frac{24}{5} \phi^{2} \frac{g(2)}{D_{0}^{s}}\langle V(2)\rangle_{2}^{0} \\
& +\frac{24}{5} \phi^{2} \frac{1}{D_{0}^{s}} \int_{2}^{\infty}\langle V\rangle_{2}^{0}(r) \frac{d g}{d r} d r,
\end{aligned}
$$

where we have specified that the potential at infinity be zero. Equation (73) shows that the weak potential makes a linear perturbation to the viscosity proportional to the value of the potential at contact; the integral in Eq. (73) should be small, especially as $\phi_{m}$ is approached. The scalings derived earlier for the behavior of the viscosity as $\phi_{m}$ is approached remain unchanged; only the numerical coefficients will be modified by adding $\langle V(2)\rangle_{2}^{0}$. Similarly, the viscoelastic response over the entire frequency range will be modified only in a quantitative way. Note that the equilibrium distribution $g(2)$ also contains an effect of the weak interparticle forces.

The more interesting case occurs when the interparticle forces result in a significant departure (at any point $r$ ) 
from the hard-sphere distribution, as occurs, for example, when there are strong repulsive forces between particles. Since the detailed response will ultimately depend upon the precise form of these forces, here we shall take a simple model in order to understand the generic effects of repulsive forces. The model is a very steep repulsive force that may be approximated as a hard-sphere-like repulsion at an interparticle separation of $r=2 b$, with $b>a$. Interactions of this form would be approximately realized with highly charged particles in a low ionic strength fluid such that the Debye length is suitably large. This model also brings into sharp focus the relative importance of the "thermodynamic," $g(2)$, versus the "hydrodynamic," $1 / D_{0}^{s}$, effects.

We shall assume an interparticle force in the form of a delta function at $r=2 b$ [cf. Eq. (12)]. This interparticle force introduces a new length into the the problem, $b$, in addition to $a$. It is easy to see that as far as the equilibrium structure is concerned it is the length $b$ and the volume fraction based on this length $\phi_{b}=4 \pi b^{3} n / 3$ that determine the structure. Thus, the osmotic pressure is given by

$$
\frac{\Pi I}{n k T}=1+4 \phi_{b} g(r=2 b),
$$

where it is the radial-distribution function at the contact value $2 b$ that enters, not at $2 a$. The contribution to the osmotic pressure from the Brownian stresses is identically zero [cf. Eq. (11)], as there are no particles at contact at $r=2 a$. The "true" radius only enters in determining the hydrodynamic interactions as they are sensitive to the actual, and not effective, volume of the particle. By the same token, the Brownian contribution to the stress $\left\langle\Sigma_{P}^{B 1}\right\rangle$ will be identically zero. When neglecting hydrodynamics (neglecting $\left.\left\langle\Sigma_{P}^{D 2}\right\rangle\right)$ all the stress comes from the interparticle potential.

Working with the hard-sphere potential at separation $2 b$ it is easy to see that the development that led to Eq. (40a) for $f$ will be unchanged if we use the length $b$ to nondimensionalize all lengths. The appropriate Peclet number now becomes $P e_{b}=\dot{\gamma} b^{2} / D_{0}$. Note, however, that the translational diffusivity is that of a sphere of radius $a$ not $b$. This is because the hydrodynamic size of the particle is given by $a$. This fact also means that when we rescale by the short-time self-diffusivity it is the short-time selfdiffusivity at the hydrodynamic or true volume fraction $\phi$, and not at the interparticle potential or thermodynamic volume fraction $\phi_{b}$. Thus, the characteristic time scale for the viscoelastic response is $b^{2} / D_{0}^{s}(\phi)$.

The solution we have obtained for $f$, Eq. (56), is the same as before, as are the expressions for the real and imaginary parts of the complex viscosity. All that changes is that we need to evaluate Eq. (71) for the interparticle force contribution. Noting that the delta function at $r=2 b$ will project out $g(2 b)$ and $f(2 b)$, we have for the zerofrequency dynamic or steady shear viscosity

$$
\eta_{P 1}=\eta_{P 1}^{\prime}(0)=\frac{12}{5} \frac{a}{b} \phi_{b}^{2} \frac{g\left(2 ; \phi_{b}\right)}{D_{0}^{2}(\phi)}
$$

The factor of $a / b$ enters because the infinite dilution diffusivity has been made nondimensional by $a$.

This important conclusion that the hydrodynamics occur on the scale of $a$ and the deformation to the structure on the scale of $b$ means that for a highly repulsive interparticle potential the steady shear viscosity should diverge as the random close packing of the thermodynamic volume fraction $\phi_{b m}$ is approached, because $g(r)$ at contact diverges as $\left(1-\phi_{b} / \phi_{b m}\right)^{-1}$. However, the short-time selfdiffusivity will not vanish at $\phi_{b m}$, and the singular form of the viscosity is predicted to be

$$
\eta_{P 1}(0) \sim\left(1-\phi_{b} / \phi_{b m}\right)^{-1} \text { as } \phi_{b} \rightarrow \phi_{b m},
$$

in contrast to the $\left(1-\phi / \phi_{m}\right)^{-2}$ behavior for Brownain hard spheres.

As mentioned earlier, the reduced viscosity functions are given by Eqs. (49)-(50) where we must add $\eta_{P_{1}}^{\prime}$, $\eta_{P 2}^{\prime}$, etc. paralleling the development for the Brownian contribution. We have

$$
\frac{\eta^{\prime}(\omega)-\eta_{\infty}^{\prime}}{\eta_{0}^{\prime}-\eta_{\infty}^{\prime}}=\frac{\eta_{P 1}^{\prime}(\omega)-\eta_{P 1}^{\prime}(\infty)}{\eta_{P 1}^{\prime}(0)-\eta_{P 1}^{\prime}(\infty)},
$$

and

$$
\frac{\eta^{\prime \prime}(\omega)}{\eta_{0}^{\prime}-\eta_{\infty}^{\prime}}=\frac{\eta_{P 1}^{\prime \prime}(\omega)}{\eta_{P 1}^{\prime}(0)-\eta_{P 1}^{\prime}(\infty)}
$$

These nondimensional functions are identical to the Brownian case with $b^{2} / D_{0}^{2}(\phi)$ replacing $a^{2} / D_{0}^{2}(\phi)$ in Eqs. (59)-(60). Similarly, the high-frequency dynamic modulus is given by Eq. $(66)$ with $(a, \phi)$ replaced everywhere by $\left(b, \phi_{b}\right)$.

Finally, the hydrodynamic viscosity is still given by Eq. (26) where the volume fraction is the hydrodynamic one $\phi$. Thus, as $\phi_{b} \rightarrow \phi_{b m}$ the hydrodynamic viscosity will not diverge because we are still far $(b \gg a)$ from $\phi_{m}$.

To summarize, for strongly repulsive interparticle forces occurring on the length scale $b$, the hydrodynamic interactions still occur on the length scale $a$ and set the value of the short-time self-diffusivity $D_{0}^{s}(\phi)$ and the hydrodynamic viscosity $\eta_{H}(\phi)$. The viscoelastic response occurs on the time scale $b^{2} / D_{0}^{s}(\phi)$ because relaxation of the local microstructure requires a particle to diffuse over the length $b$ not $a$. The appropriate Peclet number is the ratio of the diffusive time to the shear time $P e_{b}=\dot{\gamma} b^{2} / D_{0}^{s}(\phi)$, and this must be small for the departures from the equilibrium behavior to be small and linear. In the absence of hydrodynamics, which is not an approximation but correct when $b \gg a$, the viscoelastic response is identical to that predicted by Eqs. (59)-(60) and shown in Fig. 2, with $b^{2} / D_{0}^{2}(\phi)$ replacing $a^{2} / D_{0}^{2}(\phi)$. The high-frequency dynamic modulus is given by the same formula as in the Brownian limit with $\left(b, \phi_{b}\right)$ replacing $(a, \phi)$, as is the lowfrequency dynamic modulus Eq. (70). What changes is the singular behavior of the viscosity as $\phi \rightarrow \phi_{b m} ; g(2)$ still diverges as before at $\phi_{b m}$ but $D_{0}^{s}(\phi)$ does not vanish and thus $\eta_{0}^{\prime} \sim \eta\left(1-\phi_{b} / \phi_{b m}\right)^{-1}$.

The fundamentally different singular behavior of the viscosity as maximum packing is approached naturally 
raises the question of how can one be certain that no shortrange repulsive forces are present in experimental dispersions, such as those of van der Werff and de Kruif ${ }^{20}$ and $\mathrm{Krieger}^{26}$ shown in Fig. 1. In other words how do we guarantee a true hard-sphere behavior at $r=2 a$ ? The answer is that if the range of the repulsive force is on the same scale as the hydrodynamic interactions, $b \approx a$, then as close packing is approached $D_{0}^{5}(\phi)$ will decrease (and decrease linearly, since $D_{0}^{s}$ is approximately linear over the entire range of $\phi$ ) and $\eta_{P 1}$ given by Eq. (75) is of the identical form as $\eta_{B 1}$ of Eq. (53). Thus, interparticle forces with $b \approx a$ will produce behavior identical to Brownian hard spheres. What is lost from the Brownian contribution to the stress by the fact that there are no particles at contact at $r=2 a$ is precisely made up from the interparticle force contribution to the stress at $r=2 b$, just as is the case for the osmotic pressure in equilibrium. This compensation between the Brownian and interparticle stresses must explain the agreement of the viscosity measurements for true Brownian hard spheres and near hard spheres and the robustness of the singular scaling $\left(1-\phi / \phi_{m}\right)^{-2}$.

\section{CONCLUSIONS}

In this paper we have determined the singular scaling of the viscosity of colloidal dispersions, predicting that the viscosity diverges at random close packing as $\eta_{0}^{\prime}$ $\sim \eta g(2 ; \phi) / D_{0}^{s}(\phi) \sim \eta\left(1-\phi / \phi_{m}\right)^{-2}$. This prediction was shown to be quite generic-insensitive to whether the particles interact as perfect Brownian hard spheres or through short-range $(b \approx a)$ interparticle forces of colloidal origin. Moreover, the singular scaling is not affected by any of the assumptions made in closing the hierarchy of particle evolution equations. Physically, the viscosity diverges at random close packing because the number of "contacting" particles becomes infinite and the short-time selfdiffusivity vanishes as the touching particles are "stuck" by the hydrodynamic lubrication forces. Each of these physical effects contributes one factor of $\left(1-\phi / \phi_{m}\right)^{-1}$.

With this knowledge and the similarity between the Brownian stress and that due to interparticle forces, one can immediately conclude that the same scaling will occur for Brownian particles that interact through colloidal forces if the range of the colloidal force is comparable to the actual (or hydrodynamic) particle size. In simpler terms, if the thermodynamic volume fraction, which sets the equilibrium structure, is comparable to the hydrodynamic volume fraction, which controls the hydrodynamic interactions, particularly the lubrication interactions, then the viscosity will diverge as $\left(1-\phi / \phi_{m}\right)^{-2}$. On the other hand, if the thermodynamic volume fraction greatly exceeds the hydrodynamic volume fraction, as occurs with long-range repulsive forces, then the viscosity diverges as random close packing of the thermodynamic volume fraction is approached with a weaker exponent coming only from $g(r)$ at contact, i.e., $\eta_{0}^{\prime} \sim \eta\left(1-\phi_{b} / \phi_{b m}\right)^{-1}$.

It would be interesting to see if this prediction is born out by experiment. It has long been known that one should use the thermodynamic volume fraction to scale rheologi- cal data, but experimentalists have tended to use a singular viscosity with an exponent of -2 . Often this has been chosen because it fits the data well over a wide range of volume fractions, not just as maximum packing is approached. A careful examination of data very near $\phi_{b m}$ should reveal whether -2 or -1 is the correct exponent.

With these simple scaling relations and the important observation that the characteristic relaxation time is the diffusive time $a^{2} / D_{0}^{s}(\phi)$, we showed that the viscoelastic response of colloidal dispersions, as expressed in terms of the dynamic viscosity or modulus, is a universal function of the reduced frequency $\omega a^{2} / D_{0}^{s}(\phi)$. Furthermore, since the dominant contribution to the stress comes from the value of the perturbed pair-distribution function at contact, we were able to make the simple approximation of neglecting hydrodynamic interactions after scaling with $D_{0}^{s}(\varphi)$ in the pair-evolution equation, which allowed us to obtain analytical expressions for both the magnitude and frequency dependence of the viscosity. These a priori predictions were shown to agree excellently with the available experimental data for all volume fractions and all frequencies.

The theory presented here forms a natural starting point for the consideration of specific interparticle force laws and their influence on suspension rheology. Qualitatively, the theory suggests that one consider both the thermodynamic and hydrodynamic volume fractions and scale the data with the reduced shear rate $\dot{\gamma} a^{2} / D_{0}^{s}(\phi)$ or reduced frequency $\omega a^{2} / D_{0}^{s}(\phi)$, with the length scale being given by the larger of the range of the interparticle forces or the particle size. For quantitative calculations, one can begin with the no hydrodynamic approximation and include the interparticle force contribution in the pair-evolution equation through derivatives of the equilibrium distribution function [cf. Eq. (40a)]. Note, that we only needed the value of $f$ at contact to predict the viscoelastic response. To predict the deformation of the structure for all $r$, which can be observed by light scattering, might require a more complete analysis of the pair-evolution equation.

The next logical step would be to include conditionally averaged hydrodynamic interactions and also compute the magnitude of the Brownian and interparticle force contributions to the bulk stress that arise from hydrodynamic interactions [Eqs. (43) and (72)]. While such an approach is feasible, experience has shown that with hydrodynamic interactions one either makes very simple approximations or a full many-body treatment is needed. The same may be true here. The closure and simple approximations used here capture the essence of the behavior, even, it seems, quantitatively. The straightforward "improvements" possible by including conditionally averaged hydrodynamic interactions, for example, may not work well.

The observation that the relaxation time is given by the short-time self-diffusivity can be carried over to the farfrom equilibrium behavior. Colloidal dispersions generally shear thin, with the magnitude of the shear thinning and its location in shear rate depending in the volume fraction. ${ }^{20}$ In a future paper ${ }^{8} \mathrm{I}$ show that one can collapse the shear thinning behavior for the steady shear viscosity far from 
equilibrium to a single universal curve by normalizing the magnitude by the steady shear viscosity at zero shear rate and using the Peclet number based on the short-time self diffusivity at the appropriate volume fraction.

It has also been noted that far from equilibrium the dispersion's structure changes significantly from the equilibrium random structure assumed here, forming layered or string-like structures. ${ }^{12,29}$ In a more ordered state, $g(2)$ will diverge at a higher volume fraction than 0.63 assumed here, and the short-time self-diffusivity will also remain finite up to a larger value of $\phi$. The viscosity will still diverge as $\left(1-\phi / \phi_{m}\right)^{-2}$, however, with $\phi_{m}$ exceeding 0.63 and being set by the "ordered" structure present at high Peclet numbers. Experiment ${ }^{20,23}$ suggests that the viscosity at steady shear rates for $P e \approx 10-100$ diverges at $\phi_{m} \approx 0.71$ with the same exponent of -2 , which is precisely as this theory predicts. What is important in the theory is the divergence of $g(r)$ at contact and the vanishing of $D_{0}^{s}$, not that this occurs at random close packing. The same scaling will hold for any $\phi_{m}$, showing once again the robustness of the singular scaling law.

As a final comment, it has often been speculated that there should be a simple relation between the effective viscosity and the selfdiffusivity. That is, the Stokes-Einstein relation gives the diffusivity as $D=k T / 6 \pi \eta a$, and one wonders if it is appropriate to replace $\eta$ with $\eta_{\text {eff }}$. The short-time self-diffusivity owes its existence and volume fraction dependence to the reduced mobility a particle experiences because of hydrodynamic interactions. Since the particle does not move a significant fraction of its size, the structure "sampled" by the particle is the equilibrium one. Thus, the natural connection to the viscosity is through the hydrodynamic viscosity $\eta_{H}$, or the high-frequency dynamic viscosity $\eta_{\infty}^{\prime}=1+\eta_{H}$, as this too is of purely hydrodynamic origin and averaged over the equilibrium structure. Furthermore, $\eta_{H}$ is singular as $\left(1-\phi / \phi_{m}\right)^{-1}$ as close packing is approached, which is consistent with the observation that $D_{0}^{s}$ vanishes linearly at close packing. Thus, the short-time self-diffusivity scales as $D_{0}^{s}$ $\sim k T / \eta_{\infty}^{\prime} a$.

The long-time self-diffusivity, $D_{\infty}^{s}(\phi)$, on the other hand, requires a particle wander far from its starting point, and in doing so it must deform the local structure. This deformation to the equilibrium structure is not unlike that caused by a weak shearing motion such as that considered here. The natural connection is then between the long-time self-diffusivity and the steady shear viscosity $\eta_{0}^{\prime}$. We expect $D_{\infty}^{s} \sim k T / \eta_{0}^{\prime} a$. Since $\eta_{0}^{\prime} \sim\left(1-\phi / \phi_{m}\right)^{-2}$ as $\phi_{m}$ is approached, the long-time self-diffusivity should vanish quadratically as close packing is approached. It is known experimentally that the long-time self-diffusivity is smaller than the short-time self-diffusivity and this estimate of its behavior at close packing is not in disagreement with experiment. ${ }^{15,16}$ In fact, the idea that there is a close analogy between the long-time self-diffusivity and the steady shear viscosity and that the deformation to the structure caused by the diffusing particle will be similar to that considered here, but will relax with the long-time self-diffusivity, can be used to develop a theory predicting the long-time self- diffusivity. ${ }^{30}$ This theory predicts that $D_{\infty}^{s}(\phi)=D_{0}^{s}(\varphi)[1$ $+2 \varphi g(2 ; \varphi)]^{-1}$, which agrees quite well with experiment and shows a very explicit relation between $D_{\infty}^{s}(\phi)$ and $\eta_{0}^{\prime}: \quad D_{\infty}^{s}(\phi) \eta_{0}^{\prime}(\phi) \sim \frac{6}{5} \phi(k T / 6 \pi \eta a)$ as $\phi \rightarrow \phi_{m}$.

\section{ACKNOWLEDGMENTS}

This work was supported in part by Grant No. CTS9020646 from the National Science Foundation.

\section{APPENDIX}

To derive Eq. (10) we write the Brownian stress, $\mathbf{S}^{B}$ in Eq. ( $8 \mathrm{~b})$, as a probability integral in the limit of large $N$ :

$$
\begin{aligned}
\left\langle\mathbf{S}^{B}\right\rangle & =-k T\left\langle\nabla \cdot\left(\mathbf{R}_{S U} \cdot \mathbf{R}_{F U}^{-1}\right)\right\rangle \\
& =-k T \frac{1}{N} \sum_{\alpha=1}^{N} \nabla_{\alpha} \cdot\left(\mathbf{R}_{S U} \cdot \mathbf{R}_{F U}^{-1}\right)_{\alpha} \\
& =-k T \frac{1}{N} \frac{1}{N !} \sum_{\alpha=1}^{N} \int\left[\nabla_{\alpha} \cdot\left(\mathbf{R}_{S U} \cdot \mathbf{R}_{F U}^{-1}\right)_{\alpha}\right] P_{N} d \mathbf{r}_{N},
\end{aligned}
$$

where $\left(\mathbf{R}_{S U} \cdot \mathbf{R}_{F U}^{-1}\right)_{\alpha}$ is the value corresponding to particle $\alpha$, and $d \mathbf{r}_{N}$ is the volume element for the $N$-particle distribution function. Now,

$$
\begin{aligned}
{\left[\nabla_{\alpha} \cdot\left(\mathbf{R}_{S U} \cdot \mathbf{R}_{F U}^{-1}\right)_{\alpha}\right] P_{N}=} & \nabla_{\alpha} \cdot\left[\left(\mathbf{R}_{S U} \cdot \mathbf{R}_{F U}^{-1}\right)_{\alpha} \boldsymbol{P}_{N}\right] \\
& -\left(\mathbf{R}_{S U} \cdot \mathbf{R}_{F U}^{-1}\right)_{\alpha} \cdot \nabla_{\alpha} P_{N},
\end{aligned}
$$

and with the aid of the divergence theorem, Eq. (A1) becomes

$$
\begin{aligned}
\left\langle\mathbf{S}^{B}\right\rangle= & -k T \frac{1}{N} \frac{1}{N !} \sum_{\alpha=1}^{N} \int\left(\mathbf{R}_{S U} \cdot \mathbf{R}_{F U}^{-1}\right)_{\alpha} \cdot \mathbf{n}_{\alpha} P_{N} d S_{\alpha} \\
& +k T \frac{1}{N} \frac{1}{N !} \sum_{\alpha=1}^{N} \int\left(\mathbf{R}_{S U} \cdot \mathbf{R}_{F U}^{-1}\right)_{\alpha} \cdot \nabla_{\alpha} P_{N} d \mathbf{r}_{N},
\end{aligned}
$$

where $\mathbf{n}_{\alpha}$ is the normal to the surface of the $\alpha$ th particle and we have assumed that the surface integrals at infinity vanish.

From low-Reynolds-number hydrodynamics, or more generally, from lubrication theory, ${ }^{31,32}$ the hydrodynamic stresslet at contact, $-\mathbf{R}_{S U} \cdot \mathbf{U}$, as the separation between the particle surfaces approaches zero, is directly proportional to the hydrodynamic force, $\mathbf{F}^{H}=-\mathbf{R}_{F U} \cdot \mathbf{U}$. This comes from the singular form of the resistance tensors at contact. Both $\mathbf{R}_{F U}$ and $\mathbf{R}_{S U}$ are singular as $1 / \xi$ as $\xi \rightarrow 0$, where $\xi=r-2 a$, corresponding to the large localized pressure requircd to pump out the viscous fluid separating the particles as their surfaces approach one another. Since $\mathbf{R}_{S U}$ gives the first moment of the force distribution, and all the singular force is localized at the point of contact,

$$
\mathbf{R}_{S U} \rightarrow a \mathbf{d} \mathbf{R}_{F U} \text { as }\left|\mathbf{r}_{2}-\mathbf{r}_{1}\right| \rightarrow 2 a,
$$

where $\mathbf{d}=\left(\mathbf{r}_{2}-\mathbf{r}_{1}\right) /\left|\mathbf{r}_{2}-\mathbf{r}_{1}\right|$ is the unit vector along the line of centers of any two particles. Now, the unit vector $\mathbf{n}_{\alpha}$ points out of the volume available to particle $\alpha$; therefore, at contact with another particle, $r=2 a$ 


$$
\mathbf{n}_{\alpha}=\mathbf{d} \text {, }
$$

and, hence,

$$
\left(\mathbf{R}_{S U} \cdot \mathbf{R}_{F U}^{-1}\right)_{\alpha} \cdot \mathbf{n}_{\alpha}=a \mathrm{dd} .
$$

Thus, taking into account the sum over identical particles, the integral over the surfaces of contact in Eq. (A2) becomes

$$
\begin{gathered}
-k T \frac{1}{N} \frac{1}{N !} \sum_{\alpha=1}^{N} \oint\left(\mathbf{R}_{S U} \cdot \mathbf{R}_{F U}^{-1}\right)_{\alpha} \cdot \mathbf{n}_{\alpha} P_{N} d S_{\alpha} \\
=-n k T a \oint \mathbf{n}_{2} \mathbf{n}_{2} P_{1 / 1}\left(\mathbf{r}_{2} \mid \mathbf{r}_{1}\right) d S_{2},
\end{gathered}
$$

where $P_{1 / 1}\left(\mathbf{r}_{2} \mid \mathbf{r}_{1}\right)$ is the probability density for finding a particle at $\mathbf{r}_{2}$ given that there is a particle at $\mathbf{r}_{1}$.

With this new representation for the Brownian contribution to the stress, Eq. (10) readily follows.

'G. K. Batchelor, J. Fluid Mech. 83, 97 (1977).

${ }^{2}$ W. B. Russel and A. P. Gast, J. Chem. Phys. 84, 1815 (1986).

${ }^{3}$ N. J. Wagner and W. B. Russel, Physica A 155, 475 (1989).

${ }^{4}$ B. Cichocki and B. U. Felderhof, Phys. Rev. A 43, 5405 (1991).

'W. B. Russel, in Particulate Two-Phase Flow, edited by M. Roco, (Butterworths, London, 1993).

6J. F. Brady, J. Chem. Phys. 98, 3335 (1993).

7J. C. van der Werf, C. G. de Kruif, C. Blom, and J. Mellema, Phys. Rev. A 39, 418 (1989).

${ }^{8}$ J. F. Brady (unpublished).
${ }^{9}$ J. F. Brady and G. Bossis, Ann. Kev. Fluid Mech. 20, 111 (1988).

${ }^{10}$ G. Bossis and J. F. Brady, J. Chem. Phys. 91, 1866 (1989).

${ }^{11}$ J. F. Brady and T. Phung, Proceedings of the First Tohwa Symposium: Slow dynamics in Condensed Matter AIP Conference Proceedings No. 256 (AIP, New York, 1992), p. 391.

${ }^{12}$ T. N. Phung, Ph.D. thesis, California Institute of Technology, 1993.

${ }^{13}$ R. T. Bonnecaze and J. F. Brady, J. Chem. Phys. 96, 2183 (1992); J. Rheol. 36, 73 (1992).

${ }^{14}$ G. Bossis, J. F. Brady, and C. Mathis, J. Colloid Interface Sci. 126, 1 (1988).

${ }^{15}$ P. N. Pusey and W. van Megen, J, de Phys. 44, 285 (1983).

${ }^{16}$ R. H. Ottewill and N. St. J. Williams, Nature 325, 232 (1987).

${ }^{17}$ R. J. Phillips, J. F. Brady, and G. Bossis, Phys. Fluids 31, 3462 (1988).

${ }^{18}$ A. J. C. Ladd, J. Chem. Phys. 93, 3483 (1990).

${ }^{19}$ C. W. J. Beenakker and P. Mazur, Physica A 126, 349 (1984).

${ }^{20} \mathrm{~J}$. C. van der Werff and C. G. de Kruif, J. Rheol. 33, 4221 (1989).

${ }^{21}$ C. W. J. Beenakker, Physica A 128, 48 (1984).

${ }^{22}$ L. V. Woodcock, Ann. N. Y. Acad. Sci. 37, 274 (1981).

${ }^{23}$ W. B. Russel, D. A. Saville, and W. R. Scholwalter, Colloidal Dispersions (Cambridge University Press, London, 1989).

${ }^{24}$ D. Quemada, Lecture Notes in Physics: Stability of Thermodynamic Systems, edited by J. Cases-Vasquez and J. Lebon (Springer, Berlin, 1982), p. 20.

${ }^{25}$ I. M. Krieger and T. J. Dougherty, Trans. Soc. Rheol. 3, 137 (1959).

${ }^{26}$ I. M. Krieger, Adv. Colloid Interface Sci. 3, 111 (1972).

${ }^{27}$ N. F. Carnahan and K. E. Starling, J. Chem. Phys. 51, 635 (1969).

${ }^{28}$ L. G. Leal, Laminar Flow and Convective Transport Processes (Butterworth-Heinemann, Boston, 1992).

${ }^{29}$ B. J. Ackerson, J. Rheol. 34, 553 (1990).

${ }^{30} \mathrm{~J}$. F. Brady (unpublished).

${ }^{31}$ D. J. Jeffrey, J. F. Morris and J. F. Brady, Phys. Fluids A (to be published).

${ }^{32}$ D. J. Jeffrey, Phys. Fluids. A 4, 16 (1992). 